\title{
An anatomically detailed and personalizable head injury model: Significance of brain and white matter tract morphological variability on strain
}

\author{
Xiaogai $\mathrm{Li}^{1}{ }^{10} \cdot$ Zhou Zhou $^{1} \cdot$ Svein Kleiven ${ }^{1}$
}

Received: 4 February 2020 / Accepted: 20 September 2020 / Published online: 10 October 2020

(c) The Author(s) 2020

\begin{abstract}
Finite element head (FE) models are important numerical tools to study head injuries and develop protection systems. The generation of anatomically accurate and subject-specific head models with conforming hexahedral meshes remains a significant challenge. The focus of this study is to present two developmental works: first, an anatomically detailed FE head model with conforming hexahedral meshes that has smooth interfaces between the brain and the cerebrospinal fluid, embedded with white matter (WM) fiber tracts; second, a morphing approach for subject-specific head model generation via a new hierarchical image registration pipeline integrating Demons and Dramms deformable registration algorithms. The performance of the head model is evaluated by comparing model predictions with experimental data of brain-skull relative motion, brain strain, and intracranial pressure. To demonstrate the applicability of the head model and the pipeline, six subject-specific head models of largely varying intracranial volume and shape are generated, incorporated with subjectspecific WM fiber tracts. DICE similarity coefficients for cranial, brain mask, local brain regions, and lateral ventricles are calculated to evaluate personalization accuracy, demonstrating the efficiency of the pipeline in generating detailed subjectspecific head models achieving satisfactory element quality without further mesh repairing. The six head models are then subjected to the same concussive loading to study the sensitivity of brain strain to inter-subject variability of the brain and WM fiber morphology. The simulation results show significant differences in maximum principal strain and axonal strain in local brain regions (one-way ANOVA test, $p<0.001$ ), as well as their locations also vary among the subjects, demonstrating the need to further investigate the significance of subject-specific models. The techniques developed in this study may contribute to better evaluation of individual brain injury and the development of individualized head protection systems in the future. This study also contains general aspects the research community may find useful: on the use of experimental brain strain close to or at injury level for head model validation; the hierarchical image registration pipeline can be used to morph other head models, such as smoothed-voxel models.
\end{abstract}

Keywords Traumatic brain injury $\cdot$ Subject-specific head model $\cdot$ Demons and Dramms image registration $\cdot$ Mesh morphing $\cdot$ Axonal strain $\cdot$ Finite element analysis

\section{Introduction}

Electronic supplementary material The online version of this article (https://doi.org/10.1007/s10237-020-01391-8) contains supplementary material, which is available to authorized users.

Xiaogai Li

xiaogai@kth.se

1 Division of Neuronic Engineering, Department of Biomedical Engineering and Health Systems, KTH Royal Institute of Technology, 14152 Huddinge, Sweden
Traumatic brain injury (TBI) is a leading cause of injuryrelated death and disability, with a devastating impact on the patients and their families (Maas et al. 2017). TBI causes a substantial threat to global public health and an enormous economic burden for the society, with an estimated number of 50-60 million new TBI cases occurring annually (Feigin et al. 2013). TBI influences all age groups, including children, adolescents, and the elderly, which can happen due to traffic accidents, sports injuries, and falls. Studies also suggest that TBI might represent an important modifiable risk 
factor for epilepsy, stroke, and late-life neurodegenerative diseases such as dementia and Parkinson's disease (Maas et al. 2017). Biomechanical studies, including experimental and computational studies, have long been carried out to understand brain injury mechanisms due to external mechanical input (Meaney et al. 2014). In particular, finite element (FE) head models have emerged as valuable numerical tools to study head injuries and aid the development of protection systems (Giudice et al. 2019; Horstemeyer et al. 2019; Madhukar and Ostoja-Starzewski 2019).

Human head models with varying levels of anatomical accuracy and modeling complexity have been developed during the recent decades, e.g., WSUBIM (Ruan et al. 1994; Zhang et al. 2001), SUFEHM (earlier called ULP) (Kang et al. 1997; Sahoo et al. 2014), KTH head model (Kleiven and von Holst 2002; Kleiven 2007; Giordano and Kleiven 2014b; Zhou et al. 2019a), UCDBTM (Horgan and Gilchrist 2003; Trotta et al. 2020), SIMon (Takhounts et al. 2003, 2008), THUMS (Kimpara et al. 2006; Atsumi et al. 2016), GHBMC (Mao et al. 2013a; Wu et al. 2019), and WHIM (earlier called DHIM) (Ji et al. 2015; Zhao and Ji 2019b; Zhao and Ji 2020). Continued efforts on model enhancement, including material model improvement, incorporating diffusion tensor imaging (DTI), brain-skull interface improvement, as well as mesh refinement, have led to updated versions compared to the original. Especially recent efforts on mesh refinement led to an average brain element size of about $1.8 \mathrm{~mm}$ in the WHIM V1.5 model (Zhao and Ji 2019a, b, 2020), while the element sizes in GHBMC (Mao et al. 2013a) and the refined THUMS (Atsumi et al. 2016) are also on the order of millimeter, being $2 \mathrm{~mm}$, and $1.2 \sim 5 \mathrm{~mm}$, respectively. However, the brains in the abovementioned models are simplified by smoothing out sulci and gyri, accompanied by a homogenous layer of outer cerebrospinal fluid (CSF). Further, the brain ventricles often lack anatomical details and also have a jagged interface connecting with neighboring brain elements; some models or earlier versions have no ventricles. Mesh simplification as such is partially attributed to the challenges for current meshing techniques, e.g., blocking technique (Mao et al. $2013 b)$ to capture anatomical details, while it is also a reasonable trade-off for computational efficiency. However, lacking these anatomical details hinders a model's capacity for studying certain localized injuries such as at sulci, gyri, and surrounding ventricles (further discussion found below). Nevertheless, these models with high computational efficiency have played important roles in improving our understanding of TBIs; some have found wide applications for improved vehicle safety and helmet design. Modeling techniques learned from these head models also pave the way for future models with higher anatomical accuracy.

To address anatomical accuracy, a voxel-based approach has been used to generate head models including detailed sulci, gyri, and ventricles (Ho and Kleiven 2009; Chen and Ostoja-Starzewski 2010; Miller et al. 2016; Ghajari et al. 2017). The voxel-based approach by converting voxels to hexahedral elements directly or with various smoothing algorithms is efficient and has been used widely for FE analysis of bone structures. However, a known concern is a less accurate peak strain/stress predicted from such models, especially on the surfaces due to jaggedness (Viceconti et al. 1998; Samani et al. 2001). Although the jaggedness could be reduced with various smoothing algorithms, e.g., (Camacho et al. 1997; Boyd and Müller 2006), with a larger smoothing factor, which, however, is at the expense of decreased element quality. Similarly, brain strains predicted from voxelbased head models may also have accuracy issues at jagged surfaces of outer CSF-brain and ventricle-brain interfaces, but the accuracy level is unknown and yet to be studied. Nevertheless, careful choice of result analysis, e.g., evaluating overall regional brain strains or strain distributions, allows such models to provide valuable insights attributed to its anatomical accuracy, such as high strains at sulci depth (Ho and Kleiven 2009; Ghajari et al. 2017), in line with an earlier experimental study (Lauret et al. 2009). Integrating neuroimaging with model-predicted brain strains has provided a possible association between mechanical response and chronic traumatic encephalopathy (CTE) (Ghajari et al. 2017). However, when brain strains at the jagged interfaces are of primary interest, models with conforming meshes capturing sulci, gyri, and brain ventricles are preferred. The jagged interfaces also hinder a reliable implementation of sliding or fluid-structure interaction (FSI). Lastly, falx and tentorium also need to be manually generated (Ho and Kleiven 2009; Miller et al. 2016), affecting its subjectspecific efficiency; some smooth-voxel model chose not to include falx/tentorium (Chen and Ostoja-Starzewski 2010).

Another technique for efficient generation of subject-specific models is by mesh morphing (also called warping). The concept has been used extensively in many biomechanics fields on different organs (Couteau et al. 2000; CastellanoSmith et al. 2001; Fernandez et al. 2004; Sigal et al. 2008; Bucki et al. 2010; Bijar et al. 2016; Park et al. 2017), fullbody models (Davis et al. 2016; Beillas and Berthet 2017; Liu et al. 2020), as well as smooth brain models (Hu et al. 2007; Ji et al. 2011, 2015; Wu et al. 2019), showing promising results. A typical procedure includes image registration (rigid/affine and/or followed by nonlinear registration algorithms), from which displacement field representing the geometrical difference between the subject and baseline mesh is obtained. Next, the displacement field is applied to morph the baseline mesh, resulting in a personalized mesh with updated nodal coordinates while remaining element connections. In general, the computed displacement field should comply with continuum mechanics conditions on motion, requiring diffeomorphic, non-folding, and one-to-one 
correspondence to avoid excessive element distortions (Bucki et al. 2010). Otherwise, without such reasonable element quality, not only an FE analysis is prevented from being carried out, also numerical accuracy is influenced. Morphing an anatomically detailed head model that has refined mesh sizes poses a higher requirement on smoothness (associated with Jacobian) of the computed displacement field, meanwhile provides an opportunity attributing to FE model's direct correspondence with neuroimaging and allows utilizing the advanced registration algorithms developed within the neuroimaging field. Therefore, although mesh morphing is efficient, one major challenge for using it to generate detailed subject-specific FE head model is how to design an image registration pipeline that leads to a displacement field representing well the inter-subject difference of local brain structures, meanwhile not cause excessive element distortions.

Note that anatomical accuracy for a head model is only one among the several factors influencing its biofidelity; other factors include material properties, representation of interactions between various intracranial components (e.g., brain-skull interface). Nevertheless, an anatomically accurate model provides a prerequisite for capturing local strains in areas of interest. An anatomically accurate model also allows subject-specific analysis of brain components with higher accuracy compared with models with coarse mesh due to its direct representation of the anatomical information based on subject's medical images, whereas coarse mesh models are made with reasonable volumetry representation but not the same millimeter accurate anatomical details. Generation of anatomically accurate and subject-specific head models with conforming hexahedral meshes remains a significant challenge based on the above literature review. Though conforming tetrahedral meshes are relatively easier to generate, it's not preferred in head models intended for studying TBIs due to known unfavorable characteristics, such as over stiffening and volumetric locking especially with incompressible material using first-order tetrahedral element; though second-order could alleviate but may lead to a larger computational cost than hexahedral meshes (Samani et al. 2001).

Despite promising progress, detailed TBI mechanisms remain largely unknown, reflected by not able to predict clinical symptoms, and individual-specific injury tolerances may explain to some extent (Rowson et al. 2018). Subject-specific models for more detailed mechanics of brain injuries are needed. Yet, how brain morphology and WM fiber tract morphology differences among individuals may influence brain injuries remain unclear, although a previous study investigated influences of brain sizes by global scaling (Kleiven and von Holst 2002) and inter-subject WM fiber tract influence by inserting subject's WM to the same generic head model (Giordano et al. 2017). Head size and shape vary significantly among individuals, as well as WM fiber tracts (Giordano et al. 2017), how these together may influence the brain strain responses are yet to be studied.

This study attempts to address the two challenges: (1) To develop an anatomically detailed head model with conforming hexahedral meshes; (2) Takes inspiration from pioneer works on mesh morphing and develop a new image registration pipeline for morphing a detailed head model. To address the first, the meshing approach used in our previous studies ( $\mathrm{Li}$ et al. 2017, 2019; Zhou et al. 2019b, 2020) is used. Especially the approach has been shown to generate a detailed elderly head model with a smooth interface between the brain and CSF, permitting a successful implementation of FSI at the ventricle-brain interface for studying periventricular injury (Zhou et al. 2020). Efforts toward the above two directions lead to the development of a Detailed and Personalizable Head Model with Axons for Injury Prediction (defined as the ADAPT head model), and equally important a hierarchical image registration pipeline for detailed subject-specific head model generation by morphing. The ADAPT head model is an anatomically detailed head model, including sulci, gyri, connecting the ventricular system with conforming mesh, and embedded with WM fiber tracts. The hierarchical pipeline integrating Demons and Dramms deformable registration leads to personalized (i.e., subject-specific) models with satisfactory element quality without further mesh repairing. The uniqueness of the ADAPT head model is the equipped pipeline that allows fast generation of detailed subject-specific models with large variations in head size/shape as well as local brain regions and lateral ventricles with competitive personalization accuracy. The research community may find the hierarchical image registration pipeline useful to morph other head models as well, such as smoothed-voxel head models.

This study is organized as below: Firstly, the development and validation of the ADAPT head model are presented. Secondly, the hierarchical image registration pipeline for personalization is described and its capacity is exemplified by generating six subject-specific head models with largely varying intracranial volumes (ICVs) and brain shapes. Personalization accuracy is quantified by DICE similarity coefficients. Lastly, we use the six subject-specific head models to study the influences of brain size/shape on brain strain response under the same concussive impact. We hypothesize that a large variation in brain strain and location may exist among subjects and could be revealed by anatomically detailed subject-specific models. 


\section{Method}

\subsection{Head model development}

The geometry of the ADAPT head model is based on reconstructions of the ICBM152 template generated from 152 healthy subjects (18-43.5 years) (Fonov et al. 2009, 2011), including T1W, T2W images, and probability maps. T1W and T2W images are segmented using an expectation-maximization (EM) algorithm together with the spatial information provided by the probability maps using the software Slicer 3D (3D Slicer Version 3.6 2010; Fedorov et al. 2012). Three-dimensional (3D) triangular surface meshes are then generated based on the segmented images and serve as input to the software Hexotic to generate all hexahedral elements using an Octree-algorithm (Maréchal 2009). The head model includes the brain, skull (compact and diploe porous bone), meninges (pia, dura, falx, and tentorium), CSF, and superior sagittal sinus (SSS) (Fig. 1). The brain is divided into primary structures of cerebral gray matter (GM) (i.e., cerebral cortex), cerebral white matter (WM), corpus callosum (CC), brain stem (BS), cerebellum GM and WM, thalamus, and hippocampus. CSF is divided into outer CSF and ventricular system including lateral ventricles, $3 \mathrm{rd}$ and 4 th ventricles connected by the cerebral aqueduct. Continuous mesh is used throughout the model, with all meshes node connected from the brain, pia, CSF, and dura to the inner skull, including all interfaces between the outer CSF and the brain near sulci and gyri. Note as the same material property is used for the entire brain, dividing it into subcomponents is mainly for post-processing purposes. Further, a maximum level of recursive partitioning on the initial octree cube is set to eight in the software Hexotic during meshing to allow capturing the complex structures of sulci, gyri, and ventricular system, resulting in the smallest element size of about $0.5 \mathrm{~mm}$ at these areas, and transits to larger-sized elements to $1 \mathrm{~mm}$ and largest about $2.5 \mathrm{~mm}$ at inner brain areas as shown in Fig. 2b. A smooth element size transition is ensured by the balancing rule implemented in Hexotic, with details found in Maréchal (2009). The total number of elements in the head model is 4.4 million hexahedral and 0.54 million quad elements. The minimum Jacobian in the brain is 0.45 . All simulations are conducted with LSDyna 971 R11 using an explicit dynamic solving method. A typical impact loading with a duration of $100 \mathrm{~ms}$ takes about $22 \mathrm{~h}$ using a massively parallel processing version of LS-Dyna with 256 CPUs.

The brain is modeled as hyper-viscoelastic material to account for large deformations of the tissue, with additional linear viscoelastic terms to account for the rate dependence. Material properties presented by Kleiven (2007) are used, which were based on careful analysis of experimental data. Pia, dura/falx/tentorium are modeled with nonlinear hyperelastic material using simplified rubber/foam based on the average stress-strain experimental data (Aimedieu and Grebe 2004; Van Noort et al. 1981). Material constants used in all parts of the head model are summarized in Table 1.
Fig. 1 The ADAPT head model with major components illustrated (upper), embedded with WM fiber tracts (lower left), and with connecting the ventricular system to the outer CSF (lower right)

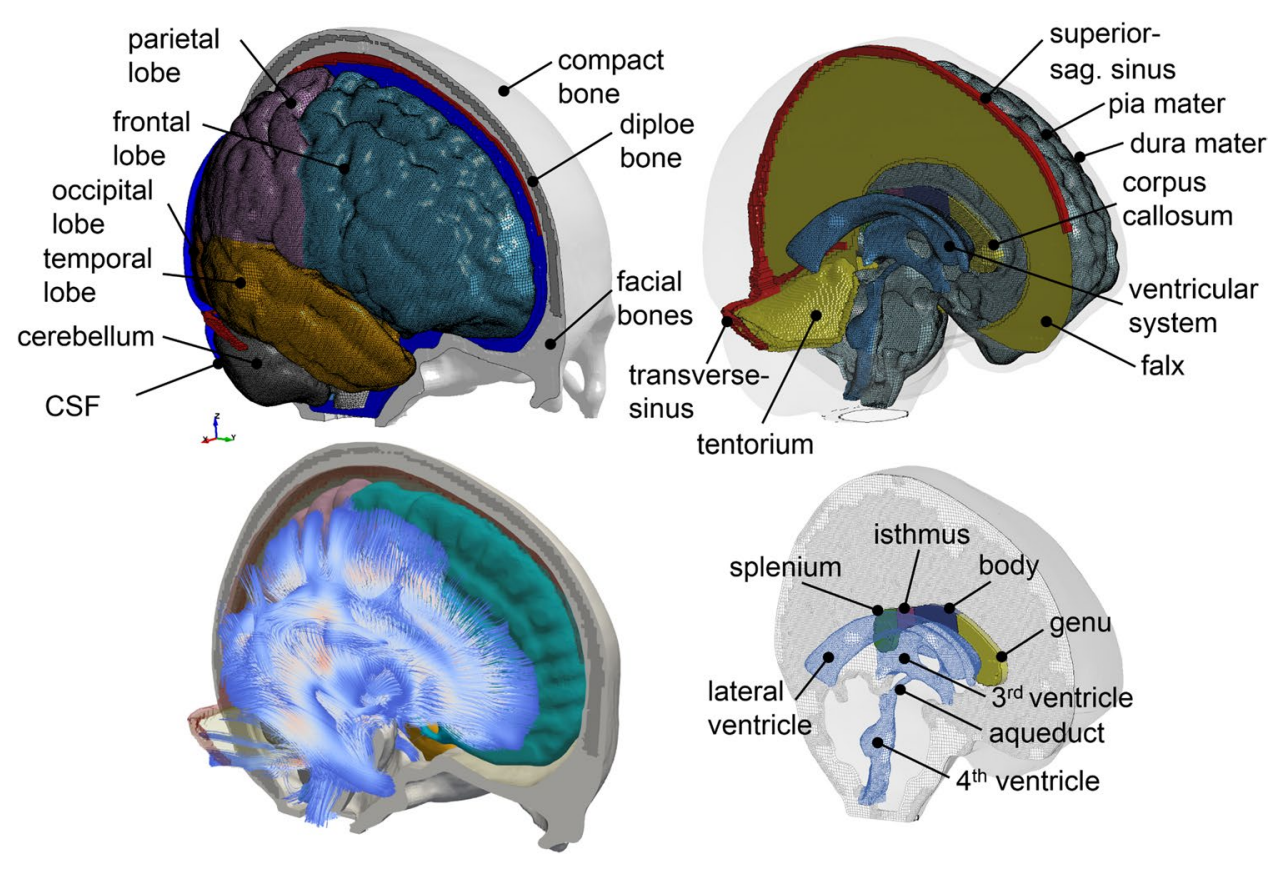


Fig. 2 Mapping DTI to the FE head model. a Brain mask image corresponding to the FE model overlay with FA calculated from DTI, showing DTI information is directly mappable to the FE model without geometrical adaption. Colorcoded FA reflects the WM orientation [red (right-left), green (anterior-posterior), and blue (superior-inferior)]. b Brain FE element with FA mapped. c View of the brain embedded with axonal fiber tracts with an enlarged image showing the axonal fibers at $\mathrm{CC}$ and BS with FA color scale indicated (a)

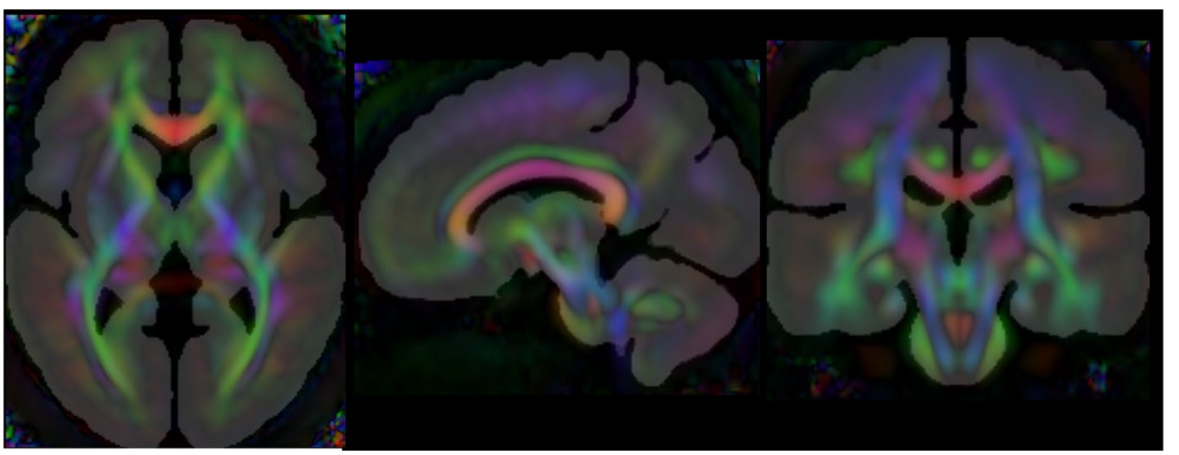

(b)

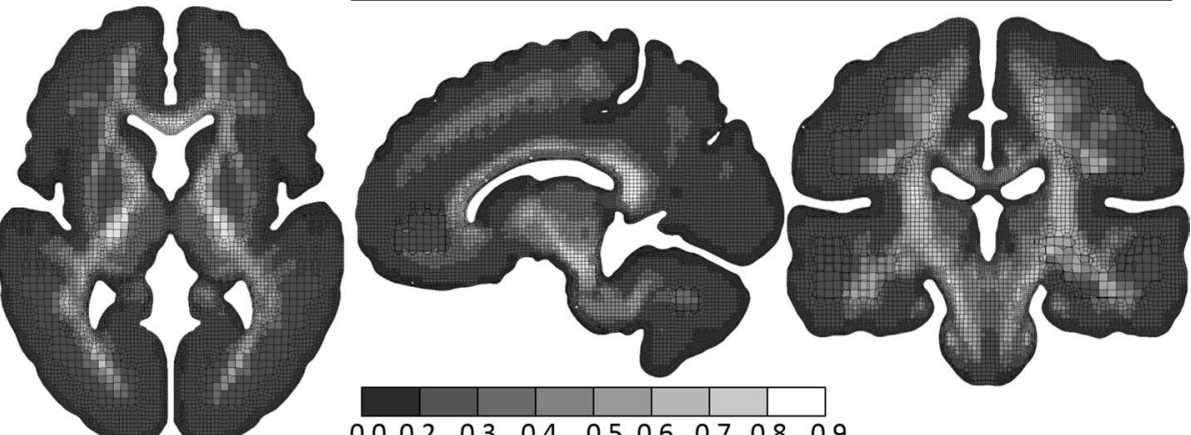

(c)

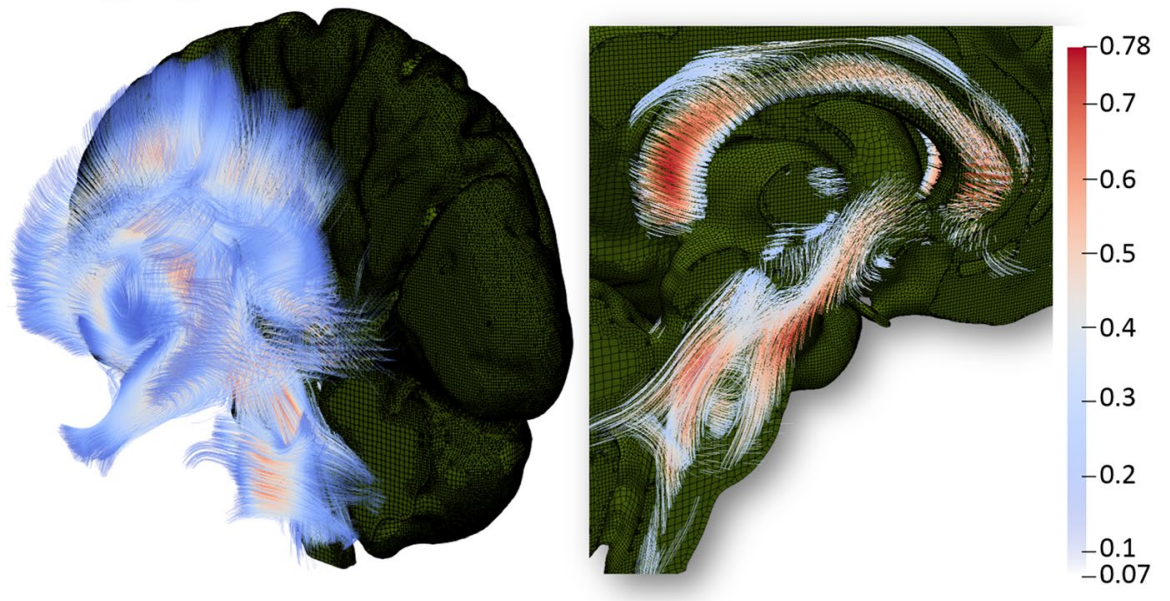

Table 1 Material properties used in the head model

\begin{tabular}{lllll}
\hline Tissue & Material constants & Density $\left(\mathrm{kg} / \mathrm{m}^{3}\right)$ & Poisson's ratio & References \\
\hline Skull compact bone & $15,000 \mathrm{MPa}$ & 2000.0 & 0.22 & (Kleiven 2007) \\
Skull porous bone & $1000 \mathrm{MPa}$ & 1300.0 & 0.24 & (Kleiven 2007) \\
Brain* & Hyper-viscoelastic & 1040.0 & $\sim 0.5$ & (Kleiven 2007) \\
CSF & $K=2.1 \mathrm{GPa}$ & 1000.0 & N/A & (Kleiven 2007) \\
Sinuses & $K=2.1 \mathrm{GPa}$ & 1000.0 & N/A & (Kleiven 2007) \\
Dura mater, falx, and tentorium & Hyperelastic & 1133.0 & N/A & (Van Noort et al. 1981) \\
Pia mater & Hyperelastic & 1133.0 & N/A & (Aimedieu and Grebe 2004)
\end{tabular}

*Ogden hyperelastic and linear viscoelastic constants used "Average" presented in Kleiven (2007) and list below: $\mu_{1}=53.8$ Pa, $\alpha_{1}=10.1$, $\mu_{2}=-120.4 \mathrm{~Pa}, \alpha_{2}=-12.9 ; G_{1}=0.31 \mathrm{MPa}, G_{2}=78 \mathrm{kPa}, G_{3}=6.2 \mathrm{kPa}, G_{4}=8.0 \mathrm{kPa}, G_{5}=1.0 \mathrm{kPa}, G_{6}=3.0 \mathrm{kPa} ; \beta_{1} \ldots \beta_{6}=10^{6} \ldots 10^{1}(1 / \mathrm{s})$, where $\mu_{i}$ and $\alpha_{i}$ are Ogden parameters, $G_{i}$ represents the shear relaxation module, and $\beta_{i}$ represents the decay constants. N/A represents not available 


\subsection{Map DTI into the head model for axonal strain calculation}

The ADAPT head model is embedded with WM fiber tracts extracted from the ICBM DTI-81 atlas (Mori et al. 2008), which contains white matter information fused with the ICBM152 template space. Eigenvalues and eigenvectors of the already calculated diffusion tensors from the ICBM DTI-81 atlas are calculated at each voxel, based on which Fractional Anisotropy (FA) and WM fiber tracts are obtained. Briefly, FA is calculated as a normalized expression of the eigenvalues, and the streamline method (Mori et al. 1999; Mori and Zhang 2006) is then used to extract WM fiber tracts associated with the 1st principal eigenvector. A more detailed description of FA and fiber tract extraction can be found in earlier studies (Li et al. 2013; von Holst and Li 2013). The calculated FA values at each voxel with a resolution of $1 \mathrm{~mm}$ are shown in Fig. 2 a.

To calculate axonal strain as defined in Eq. 1, DTI information needs to be mapped to the FE head model. As the geometry of the ADAPT head model is based on the same template as DTI, diffusion tensors from the ICBM DTI-81 atlas are directly mappable to the FE head model without geometrical adaption. Briefly, the DTI voxel closest to the centroid of each FE element is identified based on their spatial coordinates, and the FA and 1st principal eigenvector for this voxel are linked to each FE element. The resultant FA mapped at FE brain resolution is shown in Fig. 2b. The final extracted WM tracts in the whole brain contain polylines aligned with the FE head model which is shown in Fig. 2c (left), from which the $\mathrm{CC}$ and $\mathrm{BS}$ fiber tracts are enlarged (Fig. 2c right). Note the embedded WM fiber tracts are not used in this study, rather diffusion tensors extracted from the subject's own diffusion-weighted imaging (DWI) are mapped directly to the subject-specific head models (see Sect. 2.5.2). Nevertheless, the paired WM fibers tracts to the baseline head model are useful for future studies when subject's DTI is not available.

Green-Lagrange strain in the direction of WM tract (abbreviated as axonal strain hereafter) is obtained by projecting the calculated strain tensor of each element extracted from LS-Dyna solver along the axonal fiber direction according to the following equation (Giordano et al. 2014):

$\varepsilon_{\text {axon }}=\boldsymbol{\epsilon}:\left\langle\mathbf{v}_{1}\right\rangle_{e l} \otimes\left\langle\mathbf{v}_{1}\right\rangle_{e l}$

where $\epsilon$ represents the Green-Lagrange strain tensor of each element in a Cartesian vector basis and $\left\langle\mathbf{v}_{1}\right\rangle_{e l}$ denotes the axonal fiber direction in the same element obtained as the 1 st eigenvector of the diffusion tensor.

\subsection{Validation performance of the head model and CORA calculation}

The performance of the head model is evaluated by comparing experimental data of brain-skull relative motion, brain strain, as well as intracranial pressure close to or at injury level. For all the selected validation experiments mentioned above, the model is scaled to match the anthropometric measurement of the cadaveric heads. To further evaluate whether the model could predict brain response under noninjurious levels in living subjects, brain-skull relative motion and brain strain are compared with the experimental displacements and strains measured in a human volunteer using tagged MRI during mild frontal impact presented in Feng et al. (2010). All details of the validation setup are presented in Supplementary Material, with a brief description provided below.

For brain-skull relative motion validation, neutral density target (NDT) displacement curves from seven representative cases from Hardy et al. (2007) are selected, including one sagittal (C288-T3), one horizontal (C380-T2), and five coronal impacts (C380-T1, C380-T3, C380-T4, C380-T6, and C393-T3). The recalculated cluster brain strains of these seven selected cases presented in Zhou et al. (2019b) are used as experimental strain data to evaluate brain strain performance of the model. Cluster brain strains from the above seven cases are chosen, with further motivation provided in Discussion. The intracranial pressure response of the head model is compared with recordings from experiment No. 37 conducted by Nahum et al. (1977).

CORA (CORrelation and Analysis, version 3.6.1) scores are calculated to assess the level of correlation between a pair of time history curves using a sub-method included in CORA, i.e., the cross-correlation method. CORA score reported in this study is calculated as $(V+G+P) / 3$ in terms of shape $(V)$, size $(G)$, and phase $(P)$, meaning equal weights for the three parts. CORA scores range from 0 to 1 with 1 indicating a perfect match. Note another sub-method included in CORA, i.e., the corridor method is excluded, and recommended settings from (Giordano and Kleiven 2016) are adopted in this study with details provided therein.

\subsection{Hierarchical image registration pipeline for mesh morphing}

The personalization approach for subject-specific head model generation is based on Demons and Dramms deformable registrations (Fig. 3a-i). First, the diffeomorphic Demons registration (Vercauteren et al. 2009) implemented in the open-source software Slicer 3D is performed between the segmented cranial masks of the baseline (corresponding to the baseline FE mesh) and the subject after being rigidly aligned using a 6 degree-of-freedom rigid 

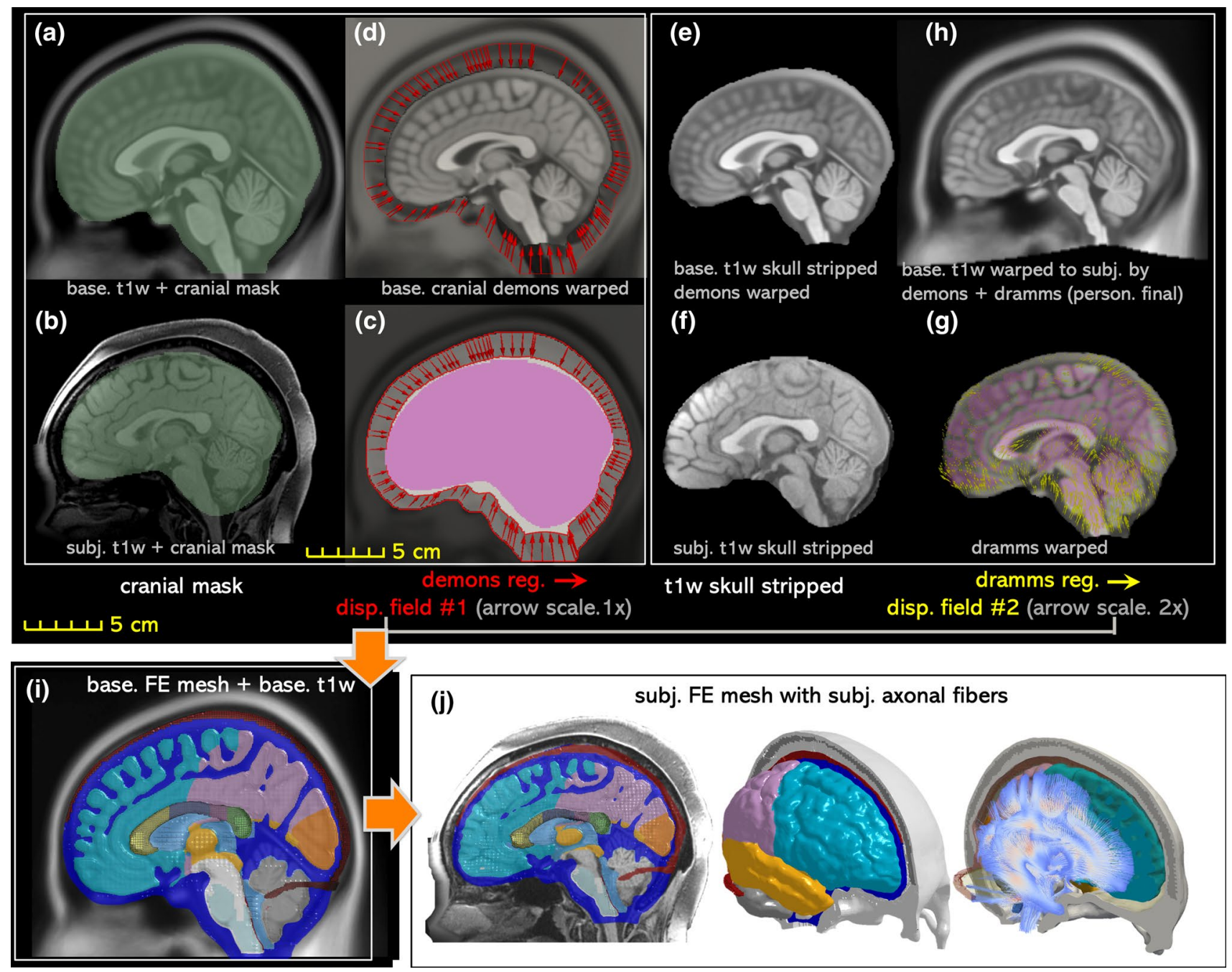

Fig. 3 The workflow of the proposed hierarchical image registration pipeline for subject-specific head model generation by morphing demonstrated with the results from the smallest female. Baseline T1W image (i.e., the T1W image corresponding to the baseline FE mesh) and the subject's T1W are segmented to obtain the cranial mask (a, b), which are used as input for Demons registration from which displacement field \#1 is obtained as indicated by the arrows (c). The inverse of Displacement field \#1 is then applied to the baseline T1W image (d), which is then skull stripped (e) and afterward together with the subject's skull stripped T1W (f) as input to Dramms registration (g). The inverse of the obtained displacement field \#2 from Dramms registration is applied to the baseline $\mathrm{T} 1 \mathrm{~W}$ and obtain the warped T1W (i.e., the T1W image corresponding to the subjectspecific mesh), which is compared with the subject's T1W to evaluate personalization accuracy. Finally, the two displacement fields add up to morph the baseline mesh (i), obtaining the subject-specific head model, including both the mesh and WM fiber tracts (j)

Sect. 2.2 resulting in a subject-specific model incorporated with the subject's own WM fiber tracts (Fig. 3j).

\subsection{Subject-specific models with axonal fibers}

The capacity of the personalization approach is demonstrated by generating six models with largely varying ICV. First, six subjects are identified by analyzing the ICVs from the WU-Minn Human Connectome Project (WUM HCP) database (Van Essen et al. 2013). For all the six subjects, both T1W and DWI images are all openly accessible. T1W sonalized model using the same procedure described in 
Table 2 Subjects selected from the HCP database representing heads of largely varying ICV

\begin{tabular}{lllc}
\hline Description & Subject ID & Age group & ICV (dl) \\
\hline Smallest (female) & 118,124 & '31-35' & 831.21 \\
5th perc. female & 568,963 & '31-35' & 1186.1 \\
50th perc. female & 771,354 & '26-30' & 1479.6 \\
5th perc. male & 185,038 & '31-35' & 1474.0 \\
50th perc. male & 172,635 & '31-35' & 1697.3 \\
Largest (male) & 223,929 & '31-35' & 2143.2 \\
\hline
\end{tabular}

The 5th percentile male and the 50th percentile female have nearly the same ICV

image is used for subject-specific mesh generation, and DWIs are mapped to the personalized model, with details presented below.

\subsubsection{Six subjects identified from HCP}

Out of the 1200 subjects from the WUM HCP database, 3T structural scans of T1W are available for 1113 subjects, and the data of ICV are already processed using the software FreeSurfer (Glasser et al. 2013). As listed in Table 2, six subjects covering a wide range of ICV are selected, including the smallest head (turns out to be a female), the largest head (turns out to be a male), and four heads with ICVs in between.

\subsubsection{WM fiber tracts extracted from DWls}

The DWIs of the six subjects with a resolution of $1.25 \mathrm{~mm}$ are processed to extract WM fibers. The downloaded DWI dataset has already been preprocessed, including corrections for gradient non-linearity, motion-correction, and eddy-currents (Glasser et al. 2013), which are further processed to extract diffusion tensors and WM fiber direction (i.e., 1st eigenvector) in each voxel using FSL v6.0.2 DTIFit with a weighted linear least squares option. The WM fiber directions are then mapped directly to the subject-specific mesh using the approach described in Sect. 2.2, based on which axonal strains are calculated.

\subsection{Evaluation of personalization accuracy}

The baseline $\mathrm{T} 1 \mathrm{~W}$ image is warped to a personalized T1W image for each subject (see "Appendix 1") using the procedure presented in Sect. 2.4. Dice coefficient (DICE) is then calculated to quantify personalization accuracy, i.e., how well the personalized T1W image (corresponding to the subject-specific mesh) reflects the subject's T1W as the ground truth. DICE is a single metric commonly used in the neuroimaging field (Bennett and Miller 2010; Zou et al. 2004) to measure the spatial overlap. DICE is defined as twice the number of elements common to both sets divided by the sum of the number of elements in each set:

$\operatorname{DICE}(A, B)=\frac{2|A \cap B|}{|A|+|B|}$

where $A$ and $B$ denote the binary segmentation labels, $|A|$ and $|B|$ are the number of voxels in each set, and $|\mathrm{A} \cap \mathrm{B}|$ is the number of shared voxels by $A$ and $B$. DICE value of 0 implies no overlap at all between both, whereas a DICE coefficient of 1 indicates perfect overlap.

To calculate the matrices, automated segmentation is performed using the software FreeSurfer (version 7.1.0) with the default brain segmentation pipeline (recon-all) for both the warped and subjects' T1W images without additional manual editing for brain regions. FreeSurfer automatically segmented whole brain and local regions of cerebral GM \& WM, CC, BS, hippocampus, thalamus, and cerebellum are used for DICE calculation, while for cranial mask and CC, DICE values are calculated based on semi-automatic segmentation by thresholding followed by noise removal, instead of using FreeSurfer segmentation due to the insufficient quality of segmented cranial mask and CC using recon-all. Further, one sagittal slice of CC is used to calculate DICE. All the labels used for matrices calculation are combined and illustrated with two subjects (Fig. 4). Note these segmented labels are only used during DICE calculation, and the quality of the automatic segmentation has no influence on the subject-specific mesh development process.

\subsection{Loading conditions and brain strain evaluation}

The six subject-specific head models are loaded with the same impact kinetics measured in a collegiate American football player resulting in loss of consciousness reported earlier (Hernandez et al. 2015). Translational accelerations and rotational accelerations (Fig. 5) are imposed on the center of gravity (C.G) of the head models.

Maximum of the 1st principal Green-Lagrange strain (MPS), and maximum axonal strain (MAS) (i.e., strains along the WM fiber direction as defined in Eq. 1) during the entire impact, as well as the locations of both metrics, are analyzed and compared between the six subjects. 


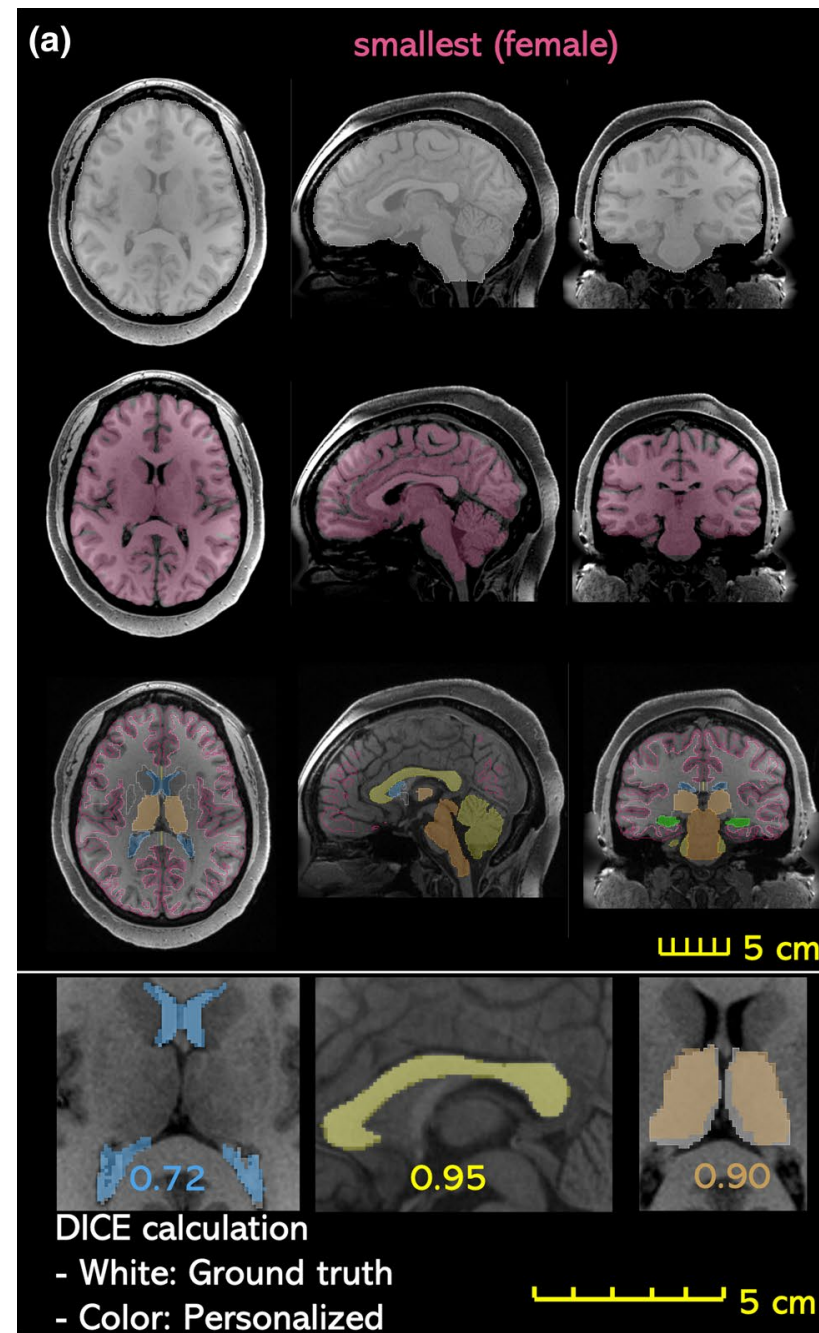

Fig. 4 Evaluation of DICE exemplified with the smallest (a) and the largest head (b), including global structures of the cranial mask (row 1), the brain (row 2), local brain regions of cerebrum GM, WM, cerebellum, hippocampus, thalamus, CC, BS, as well as lateral ventricles. T1W image of the subject is overplayed with the segmented regions from the personalized T1W image (row 3). Enlarged figures show

\section{Results}

\subsection{Validation performance of the ADAPT head model}

\subsubsection{Brain-skull relative motion}

The CORA scores for the ADAPT head model on brain motion are presented in Table 3 in comparison with head models previously developed at the same research group, including the original KTH head model (Kleiven 2007) and its updated version with FSI for brain-skull interface (Zhou et al. 2019a) interface (referred to as KTH-FSI model), as well as KTH detailed head model (Zhou et al. 2019b). Note

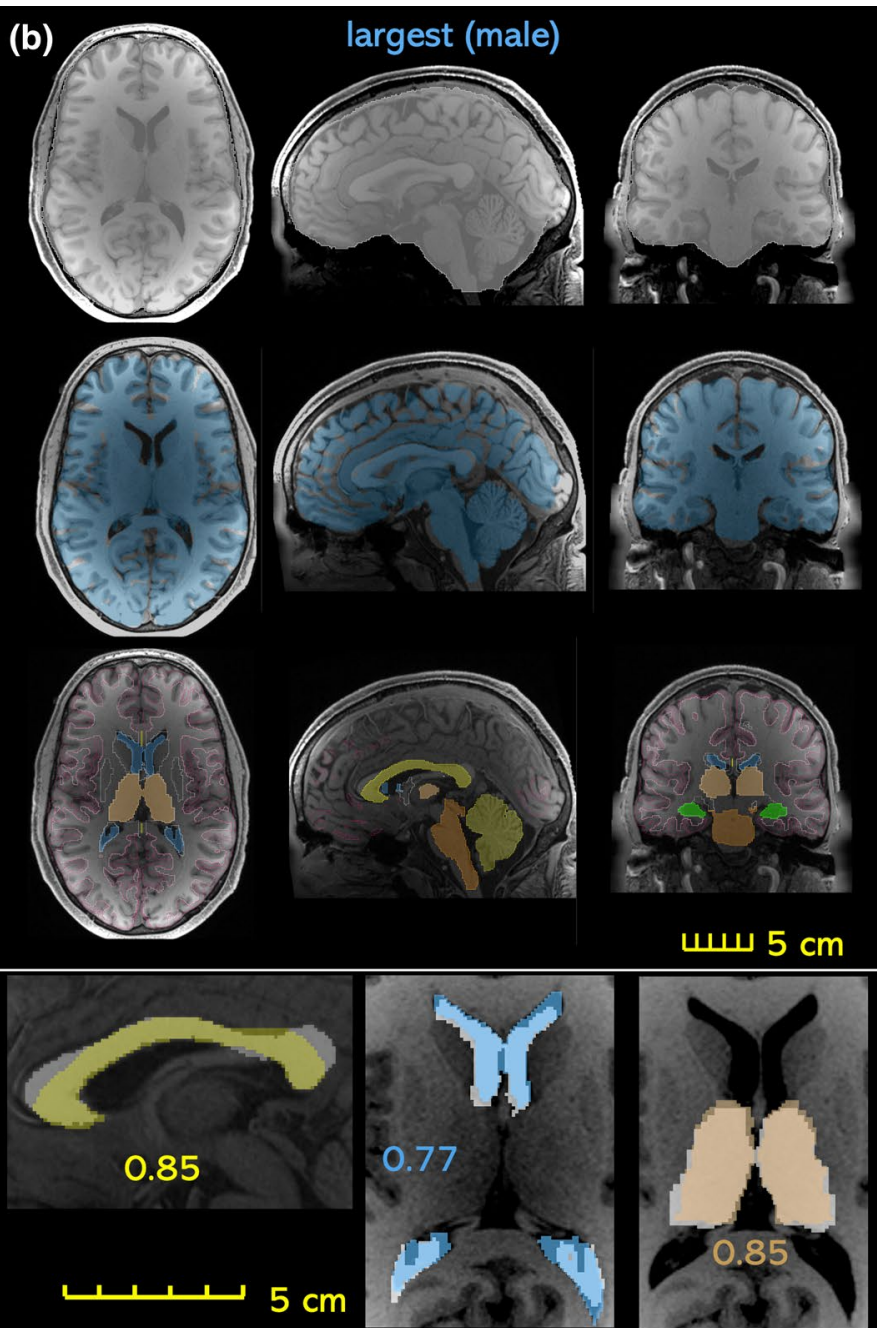

the segmented regions from the personalized T1W image (shown in color) are overlayed with the segmented regions from the subject's T1W as ground truth (gray), based on which DICE is calculated as exemplified for lateral ventricles, corpus callosum, and thalamus with DICE values shown (row 4)

that, to make the validation performance of different models amenable to a direct comparison, the CORA scores for previous models reported here are either newly calculated or recalculated using exactly the same approach and CORA settings described in this study. The CORA scores for the ADAPT head model are higher than the original KTH head model for all the seven cases, resulting in a higher mean CORA score being 0.617 versus 0.493 , respectively, while CORA scores are comparable with the KTH-FSI model for the two cases evaluated (C288-T3, C380-T4). The mean CORA score for the ADAPT is slightly lower than the KTH detailed head model (mean CORA score 0.655). To further compare predictions between the ADAPT and the original KTH head model, NDT curves of brain-skull relative 
Fig. 5 Translational (upper left) and rotational accelerations (upper right) loaded to the six subject-specific head models. All the six models are subjected to the same loading, and the head positions during the impact in two subjects are illustrated (lower row), with the smallest female in pink color and the largest male in transparent blue (dark stripes relating to rendering only)
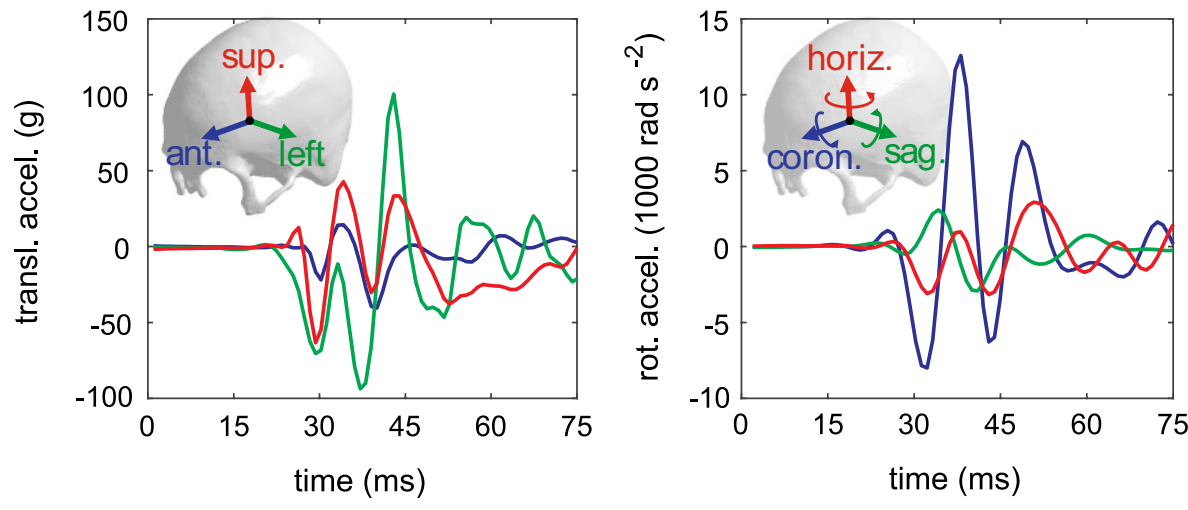

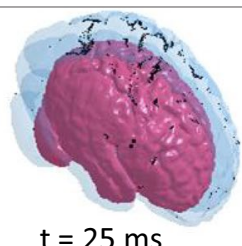

$\mathrm{t}=25 \mathrm{~ms}$

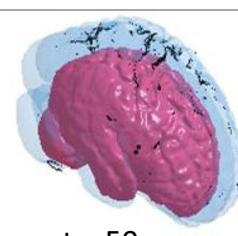

$\mathrm{t}=50 \mathrm{~ms}$

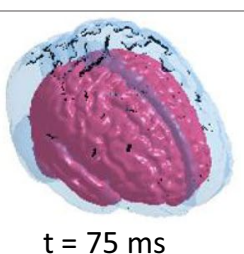

Table 3 CORA scores for the ADAPT model on brain-skull relative motion in comparison with previous models

\begin{tabular}{|c|c|c|c|c|}
\hline $\begin{array}{l}\text { Exp. cases }^{\mathrm{a}} \\
\text { (Hardy et al. 2007) }\end{array}$ & ADAPT (this study) & $\begin{array}{l}\text { Original KTH head } \\
\text { model }^{\mathrm{b}} \\
\text { (Kleiven 2007) }\end{array}$ & $\begin{array}{l}\text { KTH-FSI } \\
\text { model }^{\text {c }} \\
\text { (Zhou et al. } \\
\text { 2019a) }\end{array}$ & $\begin{array}{l}\text { KTH detailed } \\
\text { head model }^{\text {d }} \\
\text { (Zhou et al. } \\
\text { 2019b) }\end{array}$ \\
\hline C288-T3 & 0.588 & 0.423 & 0.540 & 0.538 \\
\hline C380-T1 & 0.694 & 0.560 & N/A & 0.749 \\
\hline C380-T2 & 0.549 & 0.416 & N/A & 0.623 \\
\hline C380-T3 & 0.650 & 0.503 & N/A & 0.677 \\
\hline C380-T4 & 0.658 & 0.551 & 0.646 & 0.721 \\
\hline C380-T6 & 0.625 & 0.511 & N/A & 0.702 \\
\hline C393-T3 & 0.555 & 0.500 & N/A & 0.573 \\
\hline Average & $0.617 \pm 0.055$ & $0.493 \pm 0.057$ & N/A & $0.655 \pm 0.079$ \\
\hline
\end{tabular}

${ }^{a}$ For each case, one CORA score is reported, which is the mean of CORA scores for all the evaluated NDTs as plotted in "Appendix 2". 'Simulations run for all the seven cases using the KTH head model

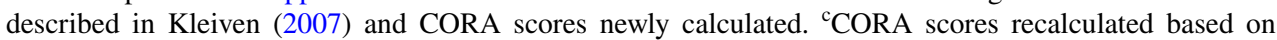
curves reported in the original study where a different CORA calculation was used. N/A: cases not run in the original study; ${ }^{\mathrm{d}} \mathrm{CORA}$ scores for the first six cases newly calculated based on curves reported in the original study as CORA scores were not reported. For case C393-T3, simulation is run for this study with CORA score newly calculated

Table 4 CORA scores for the ADAPT model on principal and shear strain

\begin{tabular}{|c|c|c|c|c|c|c|c|c|}
\hline \multirow{2}{*}{$\begin{array}{l}\text { Exp. clusters } \\
\text { (Zhou et al. 2019b) }\end{array}$} & \multicolumn{4}{|c|}{ ADAPT (principal strain) } & \multicolumn{4}{|c|}{ ADAPT (shear strain) } \\
\hline & $V$ (shape) & $G($ size $)$ & $P$ (phase) & CORA score & $V$ (shape) & $G($ size $)$ & $P$ (phase) & CORA score \\
\hline C288-T3 C1 & 0.947 & 0.361 & 0.828 & 0.712 & 0.956 & 0.426 & 0.935 & 0.772 \\
\hline C380-T1 C1 & 0.977 & 0.924 & 0.721 & 0.874 & 0.975 & 0.940 & 0.721 & 0.879 \\
\hline C380-T2 C1 & 0.986 & 0.378 & 0.935 & 0.766 & 0.983 & 0.542 & 0.935 & 0.820 \\
\hline C380-T3 C1 & 0.982 & 0.293 & 0.999 & 0.758 & 0.980 & 0.396 & 0.935 & 0.770 \\
\hline C380-T4 C1 & 0.979 & 0.249 & 0.935 & 0.721 & 0.979 & 0.317 & 0.935 & 0.744 \\
\hline C380-T6 C1 & 0.977 & 0.235 & 0.828 & 0.680 & 0.981 & 0.308 & 0.721 & 0.670 \\
\hline C393-T3 C1 & 0.934 & 0.561 & 0.999 & 0.832 & 0.925 & 0.462 & 0.935 & 0.774 \\
\hline Average & & & & $0.763 \pm 0.069$ & & & & $0.776 \pm 0.064$ \\
\hline
\end{tabular}


motion for three representative cases (sagittal C288-T3, horizontal C380-T2, coronal impact C380-T1) comparing with the experimental data (Hardy et al. 2007) are presented in "Appendix 2". Curves for all the remaining NDTs predicted from the ADAPT head model in comparison with experimental data are presented in Supplementary Material. Using the brain-motion-based CORA scores, the ADAPT head model would be rated as "fair" according to the same rating scale used earlier (Zhao and Ji 2020).

\subsubsection{Brain strain}

The mean CORA scores for the ADAPT head model on principal and shear strain for the seven evaluated clusters are 0.763 and 0.776 , respectively (Table 4). The CORA scores are compared with the KTH detailed head model (the only model so far that has used the same strain data to systematically evaluate the strain predictability of FE head model according to the authors' knowledge) (“Appendix 2", Table 8), showing comparable values.

The predicted brain strain curves from the ADAPT model are compared with the experimental brain strain data presented in Zhou et al. (2019b) (Fig. 6). Despite a large difference in the peak between the model predicted and experimental data (except for C380-T1 C1 which is closer), the shape and phase show good match, reflected by the high values of $V$ and $P$; close to 1 in some cases (C380-T3 C1, C393-T3 C1 principal strain) (Table 4). Further, the simulated brain strains are consistently lower than the experimental strain in all the seven evaluated clusters except for C380-T1 C1. More clusters are to be studied to see if the same trend holds for all the 15 cluster brain strains presented in Zhou et al. (2019b). Further discussion on brain strain validation performance and the implications are found in Discussion.

The mean CORA scores for brain strain are higher than brain-skull relative motion, attributed to high scores in shape and phase compensating the low values in size $(G)$. Using the brain-strain-based CORA scores, the ADAPT head model would be rated as "good" according to the same rating scale used earlier (Zhao and Ji 2020).

\subsubsection{Intracranial pressure and in vivo strain comparison}

The CORA scores for the ADAPT head model on intracranial pressure are presented in Table 5, with all timepressure curves presented in Supplementary Material. Note

Table 5 CORA scores of the ADAPT head model on intracranial pressure

\begin{tabular}{lll}
\hline Experimental data & Cases & Cora score \\
\hline Intracranial pressure (Nahum et al. 1977) & Frontal & 0.922 \\
No. 37 at three locations & Parietal & 0.995 \\
& Fossa & 0.859 \\
\hline
\end{tabular}
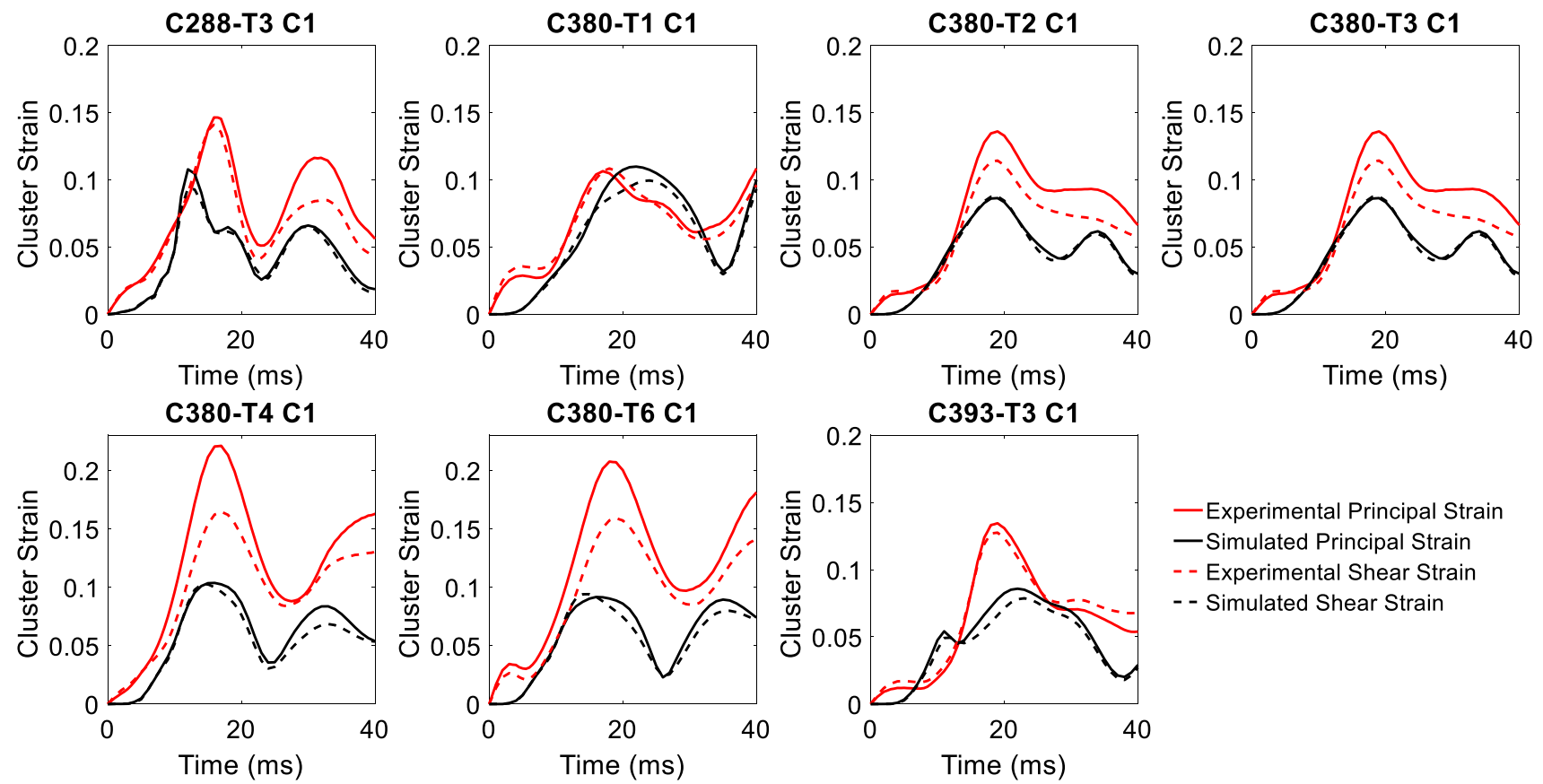

-Experimental Principal Strain

- Simulated Principal Strain

- - Experimental Shear Strain

- - Simulated Shear Strain

Fig. 6 Comparison of the ADAPT model-simulated and experimental strain. Experimental strain denotes the recalculated cluster strain presented in Zhou et al. (2019b) based on the original brain-skull relative motion experimental data from Hardy et al. (2007) 
the relatively high CORA scores for pressure should not be taken as an overinterpretation of model performance, as multiple studies have shown for an FE model with continuous mesh, brain pressure is uniquely determined by brain mass, brain shape, and linear acceleration due to its near incompressibility (Bradshaw and Morfey 2001; Zhao and Ji 2016), as done in cadaveric experiments (Nahum et al. 1977). Nevertheless, a comparison with experimental pressure data may still benefit and serve as additional verification purposes, though it's important to note brain pressure is less relevant than brain strain for blunt impact simulation.

The qualitative comparison of brain-skull relative motion and brain strain distribution with in vivo experimental data (Feng et al. 2010) (presented in Supplementary Material) indeed shows that the current ADAPT model is not capable of predicting the chosen in vivo measurement. Thus, the comparison shouldn't be interpreted as the ADAPT model has been validated against such data, rather, to highlight the need and serves as a basis for future investigation.

\subsection{Personalization accuracy evaluation of DICE for the six subject-specific models}

The boxplot of the DICE values of the cranial, the brain, and local brain regions is presented in Fig. 7 (the DICE values are listed in Table 6 in "Appendix 1"), in general showing quite good results even for $\mathrm{CC}$ with large variations between the baseline and subjects. Especially an average DICE of $0.975,0.90$, and 0.76 is achieved for the cranial mask, the brain, and hippocampus, comparable or even higher than some algorithms used in the neuroimaging field (Ou et al. 2014) for capturing inter-subject differences. DICE values for local brain regions, as well as lateral ventricles, are all above 0.6 , indicating the internal brain structures of the subject-specific head model reflect the subject to an acceptable level.

\subsection{Subject-specific head model mesh quality}

In the six subject-specific head models generated as shown in Fig. 14 in "Appendix 1", most brain elements $(95.5 \% \pm 1.2 \%$ on average for the six subjects) have a Jacobian over 0.5 , and almost all elements $(99.9 \% \pm 0.1 \%)$ have a Jacobian over 0.45 . The minimum Jacobian in the six head models is all above 0.2 . In this study, the mesh quality is considered to be satisfactory when at least $95 \%$ of the elements have a Jacobian over 0.5 . A summary of brain mesh element qualities is listed in Table 7 ("Appendix 1").

\subsection{Magnitude and location of MPS in the cerebral cortex}

The time-history curve of MPS in the cerebral cortex is extracted for each subject (Fig. 8a). Interestingly, the smallest female shows MPS occurring at $36 \mathrm{~ms}$, slightly different from other subjects except for the largest male occurring at $56 \mathrm{~ms}$ (Fig. 8a). Note the delay in the peak between both curves is mainly caused by the location difference where maximum strain occurs during the entire impact. The MPS in the cerebral cortex for all subjects is located at the sulci regions, as exemplified with results from two subjects (Fig. 8b). Additional animations of the brain strain response during the entire impact for both subjects are provided as Supplementary Videos.

The results show, in general, smaller heads tend to have a lower brain strain under the same impact loading; the lowest MPS (MPS 0.4794) and highest MPS (0.6144) are found in
Fig. 7 Boxplot of DICE values calculated for the six subjects with regions evaluated shown on the right, including the cranial mask and the brain, local brain regions, and lateral ventricles. The boxplots show the median, minimum, and maximum value

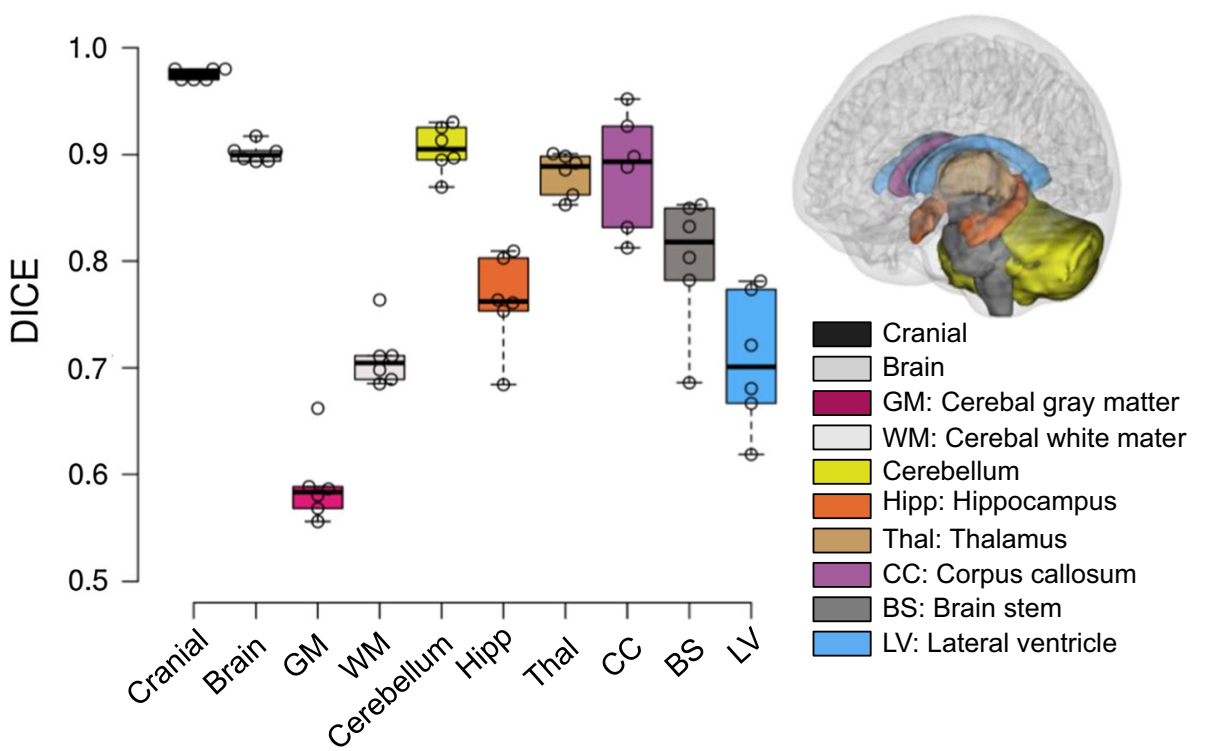


Fig. 8 a Time-history curves of MPS in the cerebral cortex during the impact of each subject. b The strain distribution at the cerebral cortex when MPS occurs exemplified with results from two subjects, captured at $36 \mathrm{~ms}$ for the smallest female and $56 \mathrm{~ms}$ for the largest male. c Locations of MPS shown by a sphere for each subject
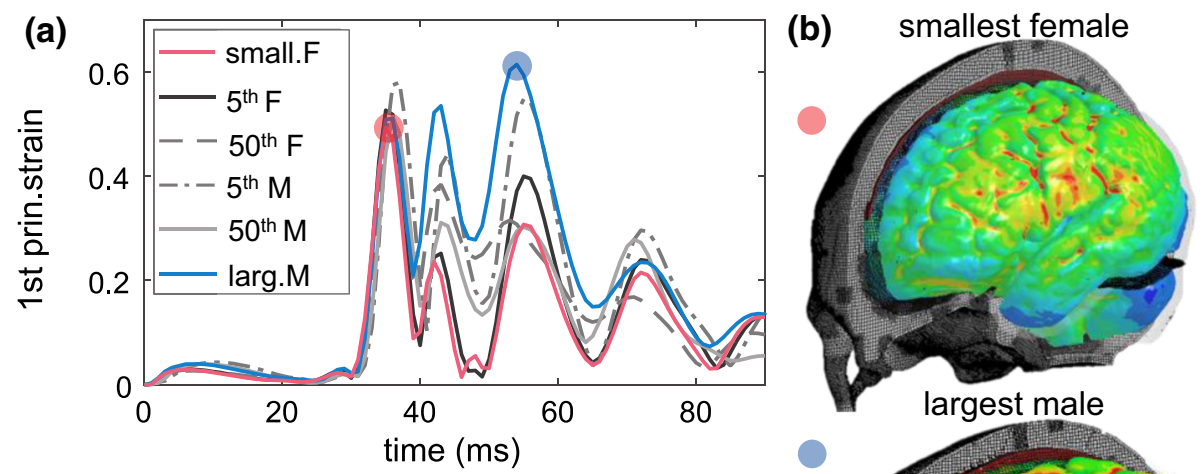

(c)
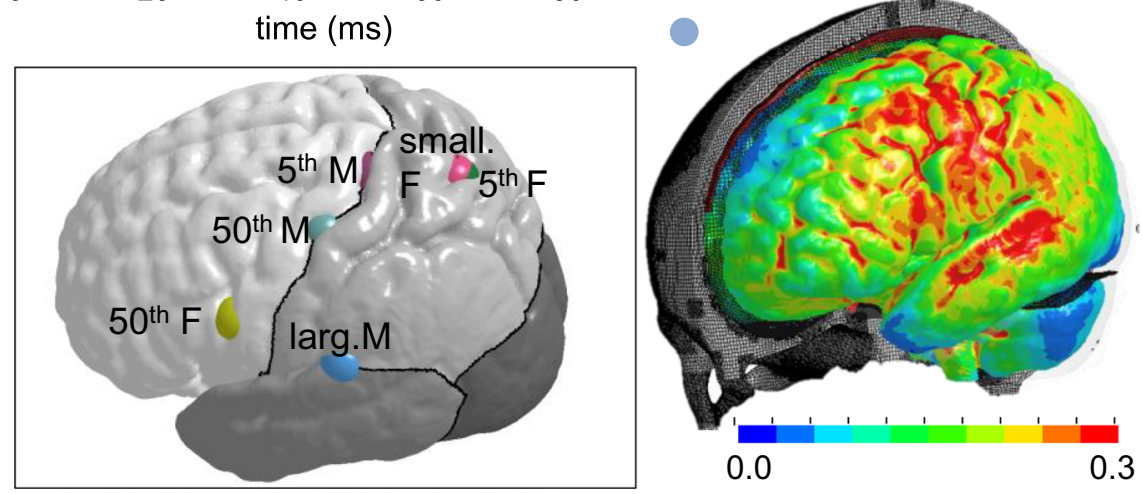
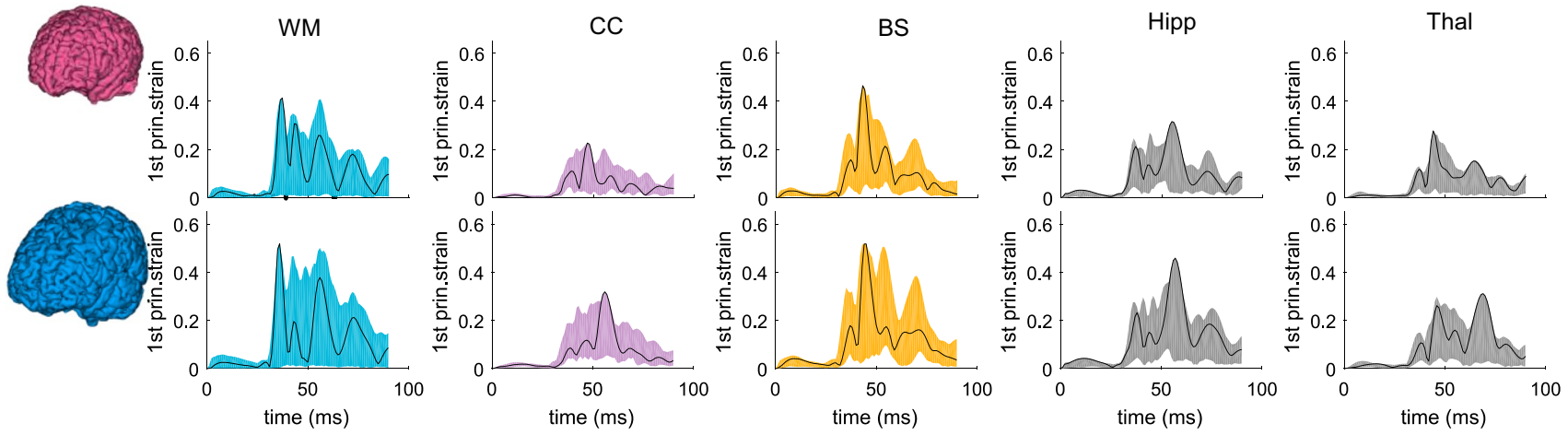

Fig. 9 Time-history curves of 1st principal G-L strain for all elements in brain regions of cerebral WM, CC, BS, hippocampus, and thalamus. The color shaded shape is formed by curves from all ele- ments for different brain regions, and the black curve shows the curve in the element with the largest strain. Upper row: smallest female. Lower row: largest male the smallest and largest head, respectively. Further, the MPS in other heads with ICV falls within these two cases but does not follow a monotonic trend. The locations of MPS for the six subjects are notably different, shifting between frontal, parietal, and temporal lobes (Fig. 8c).

\subsection{Brain regional response analysis: MPS and MAS}

The time-history curves of the 1st G-L principal strain from all elements of cerebral WM, CC, BS, hippocampus, and thalamus are evaluated and exemplified with the results from the smallest and largest heads (Fig. 9). The color shaded shape is formed by strain-time history curves from all elements, of which the curve from the element with maximum strain is plotted with black color line for each brain region. Of interest note that MPS typically occurs at a similar time between both subjects in most brain regions (i.e., similar phase in black color line between two models), except for $\mathrm{CC}$ and thalamus (Fig. 9, 2nd and 5th column), shifting to a later time in the largest head compared with the smallest head. The difference in the shaded shape indicates elementwise different MPS response between the two models, also indicate a different location of MPS. For example, the shade shape for $\mathrm{CC}$ and thalamus are notably different between the two subjects, indicating the largest strain likely occur at a different element (location) between the two models at CC and thalamus (similarly as observed for strain at cerebral cortex as shown in Fig. 8b). 


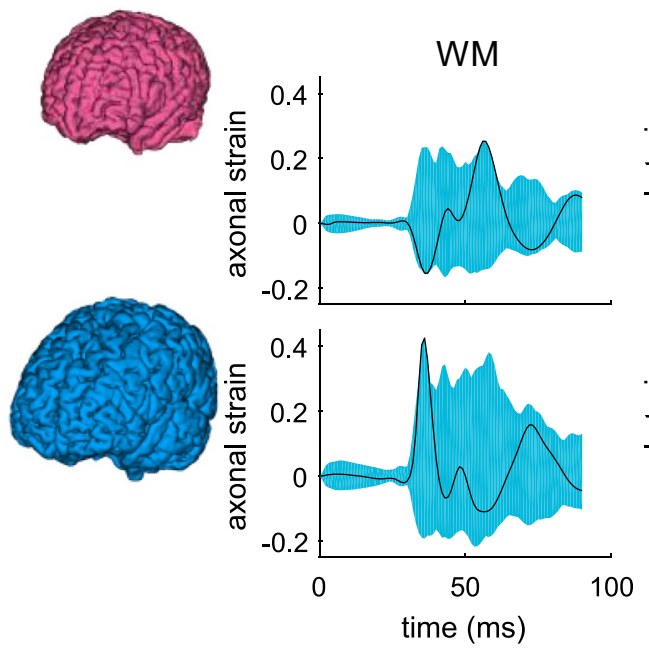

Fig. 10 Time-history curves of axonal strain for all elements in brain regions of cerebral WM, CC, and BS. The color shaded shape is formed by curves from all elements for different brain regions, and
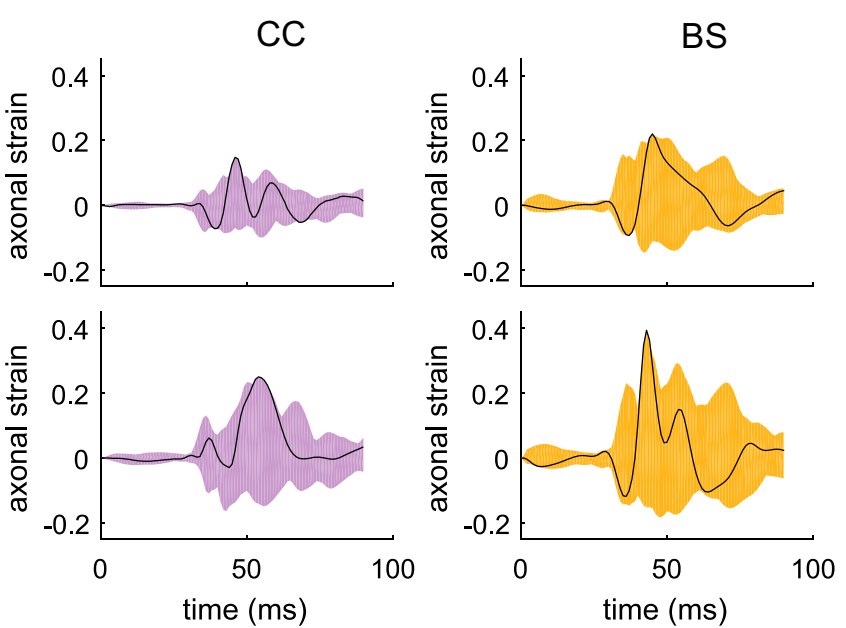

Fig. 11 Boxplots of MPS and MAS at brain regions for the six head models. MAS shows a more extensive spread than MPS, indicating a larger intersubject variability. The boxplots show the median, minimum, and maximum value
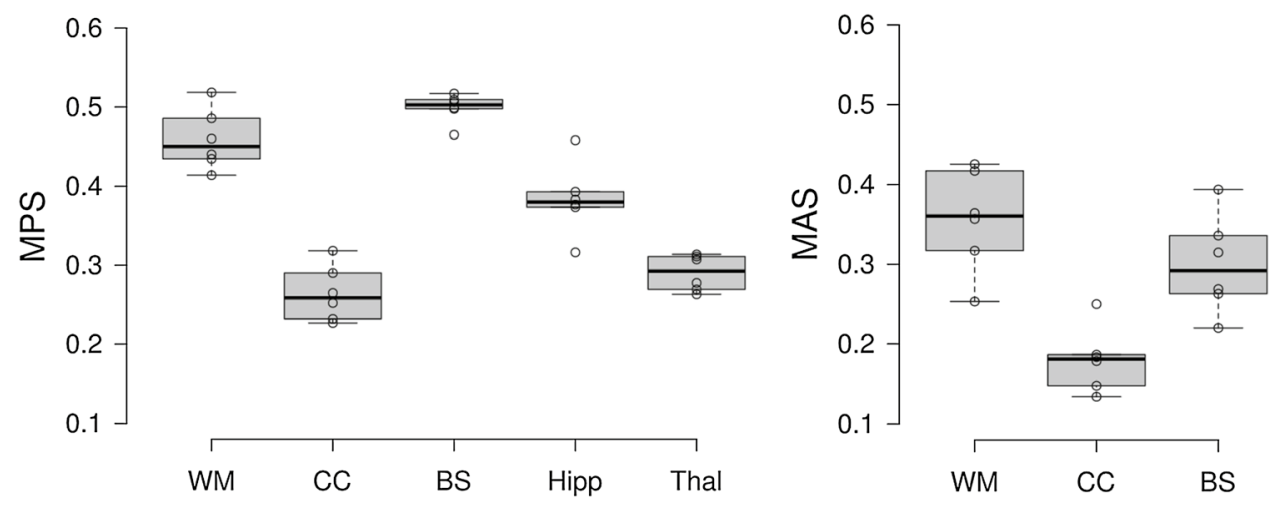

The results for axonal strain are plotted in Fig. 10 with time-history curves of axonal strain in all elements of cerebral WM, CC, BS plotted. Note axonal strain for the thalamus and hippocampus is not evaluated (grey matter region with less anisotropy), thus, is not plotted. Similarly, the color shaded shape varies between the two subjects among brain regions, indicating element-wise different MAS response between the two models. The MAS in cerebral WM occurs much later in the smallest female than in the largest male (Fig. 10, 1st row), a different trend than observed for MPS with maximum strain occurs at a similar time for WM (Fig. 9, upper row).

The time-history curves of all elements for only two subjects are presented above, and for all other subjects, only the maximum values (MPS and MAS) are presented in the boxplot (Fig. 11) (exact values are listed in "Appendix 3"). Both the MPS and MAS show significant differences among the six subjects using the one-way ANOVA test $(\mathrm{p}<0.001$ for both MPS and MAS). A similar trend is observed in the cerebral cortex that a smaller brain tends to have a smaller the black curve shows the curve in the element with the largest strain (upper row: smallest female; lower row: largest male)
MPS though not following a monotonic trend. In particular, for CC, the largest male has the largest MPS (0.32), compared with the smallest female with the lowest MPS (0.23), differing $40.3 \%$. Of the evaluated brain regions, up to $44.9 \%$, inter-subject differences in MPS are found in the hippocampus. For MAS, up to $86.21 \%$ difference in found in $\mathrm{CC}$ (the 50th percentile female with the lowest MAS and the largest male with highest MAS). The results show an even larger inter-subject variability in MAS than MPS, which is logical as WM differences are further accounted for when calculating MAS.

\subsection{Brain shape influence}

The 50th percentile female and 5th percentile male have very close ICV, and brain volumes are also similar (5\% difference). Thus, it would be interesting to analyze further to understand how brain strains vary between subjects with similar ICV but different shapes. Simulation results from the two subjects are compared, showing MPS in the cerebral 
Fig. 12 Brain strain response in the two subjects with almost the same ICV. a The time-history curve of MPS in the cerebral cortex. b Coronal and sagittal cross sections of the 1 st principal G-L strain captured at MPS occurring time in both subjects. c The time-history curve of MPS in CC. d 1st principal G-L strain distribution captured at the MPS occurring time in the respective subject (a)

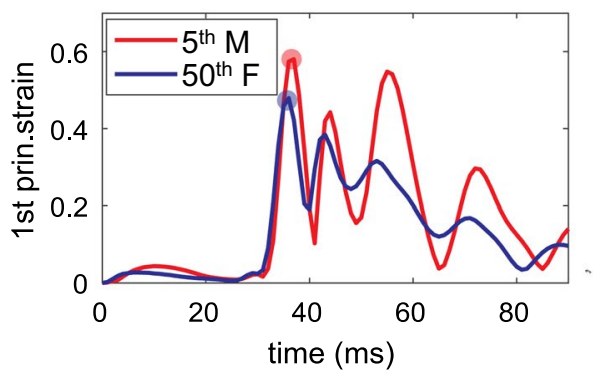

(b)

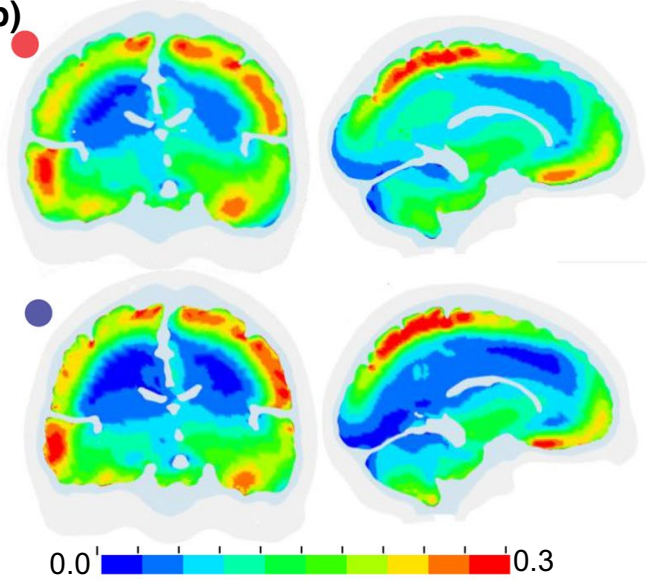

(c)

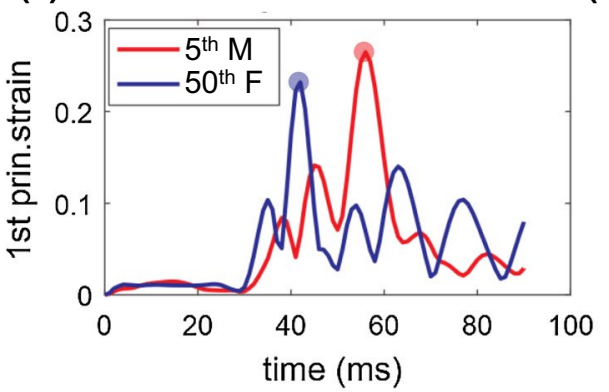

(d)

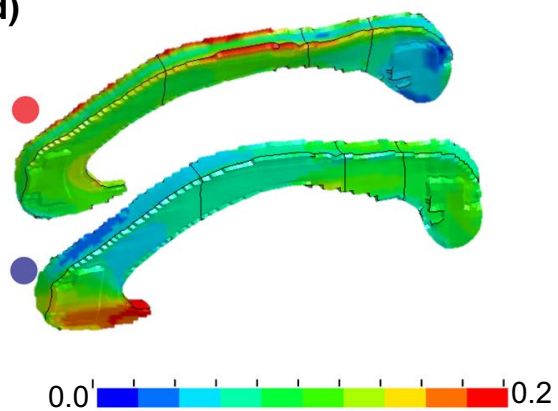

cortex differs by $14.2 \%$ (Fig. 12a), occurring at a quite different location as shown in Fig. 8c, despite a similar pattern in strain distribution (Fig. 12b). For CC, not only the MPS value differs between the two, more importantly, the occurring time (Fig. 12c), and consequently, the strain distribution pattern when MPS occurs (Fig. 12d). MPS is located at the mid-body of $\mathrm{CC}$ in the 5 th percentile male and located at the anterior in the 50th percentile female. The results provide evidence that for heads with similar ICV, the magnitude of MPS, location, and strain pattern can vary significantly due to head shape difference.

\section{Discussion}

This study presents an anatomically detailed and personalizable head model with WM tracts embedded (the ADAPT head model), and a hierarchical image registration pipeline for subject-specific head model generation by mesh morphing. The model is validated against experimental brain motion and brain strain close to or at injury level, as well as intracranial pressure, showing overall CORA score comparable or higher than earlier models. The developed pipeline allows efficient generation of anatomically detailed subjectspecific head models with satisfactory element quality. Subject-specific head models generated using this approach are shown to capture well the subjects' head geometry for the six subjects of largely varying ICVs, both on a global level (cranial mask and the brain), and local brain regions as well as lateral ventricles. Brain strains of MPS and MAS show significant differences among the six subjects due to head/ brain size \& WM morphology variability, motivating the necessity for using subject-specific head models for evaluating brain injuries.

\subsection{Head size influence on brain strain response}

The significant difference in both MPS and MAS magnitude and locations among the subjects (Figs. 8, 9, 10 and 11) seems to suggest ICV as a dominant factor influencing brain strain. Further, heads with larger ICV tend to have increased brain strains under the same loading, though not necessarily follow a monotonic increasing trend. Notably, the two subjects with very similar ICV show quite different strain patterns (Fig. 12), indicating head/brain shape may also be an important factor influencing brain strain response. It will be interesting to investigate in the future to clarify whether size or shape is more important than the other influencing brain mechanical response. For example, by using principal component analysis (PCA) (Wold et al. 1987) with more brain images, which may also allow identifying the characteristics of brain shape and WM morphologies that are most vulnerable to impact. 
Percentile strain (e.g., 95th percentile MPS) have been used in previous studies both for adults (Miller et al. 2019; Panzer et al. 2012; Wu et al. 2020; Wu et al. 2020; Beckwith et al. 2018) and children head models (Li and Kleiven 2018) to avoid potential numerical issues (e.g., strain concentration). This study uses MPS (i.e., 100th percentile MPS) to compare between subjects. Using MPS also allows identifying the element with maximum strain and plotting its time-history curves, as presented in Fig. 8a, which is from a brain element on the cortical surface. A visual check shows the strain in the plotted element has no abrupt difference comparing with its neighboring elements at the cerebral cortex (i.e., sulci/gyri) attributed to the conforming mesh, giving some confidence in the plotted curves, as well as the identified varying locations among subjects (Fig. 8c). Figure 9 with strain curves plotted from all elements at brain regions also shows no curves jumping outside, brings further confidence on the reported MPS. Finally, the time-history strain curves shown in Fig. 10b from one element in the CC, despite the non-smooth interface between $\mathrm{CC}$ and neighboring brain elements due to the same brain material used, no abrupt strain differences are observed in the plotted element comparing with its neighboring elements.

Influences of head/brain size on brain mechanical response have been studied in the past. In a $2 \mathrm{D}$ numerical study by Prange et al. (1999), coronal rotational accelerations were applied to evaluate brain strain response due to brain size differences between adults and children. Kleiven and von Holst (2002) by globally scaling a 3D adult FE head model to six heads of varying dimensions showed brain response increased almost monotonically from the smallest to the largest head under a linear acceleration. Similarly, as revealed in this current study, a larger ICV (relating to larger brain mass) tends to have a larger strain under the same impact, which is also suggested by Holbourn's scaling principle (Ommaya et al. 1967), and more recent work by Wu et al. (2020) and Panzer et al. (2014). Of interest would be to investigate whether brain strains predicted from FE models follow or can be predicted by the acceleration-mass scaling law, which indeed has been studied by Prange et al. (1999). Their results demonstrated that the mass scaling relationship was not sufficient to produce brain strain distributions. Similarly, the models in the current study incorporating sulci and gyri pose even greater challenges for such scaling laws to relate MPS with brain masses. The anatomically detailed subject-specific head models representing individual brain structural differences appear to be critical for revealing the new insights on brain size/shape influence (Figs. 8 and 12) comparing with models that have smooth brain surface and by global scaling (Kleiven and von Holst 2002).

\subsection{Image registration pipeline for mesh morphing}

Image registration is a well-developed research area in the neuroimaging field, with algorithms ranging from global (e.g., rigid align, affine) to deformable registration, which allows obtaining dense displacement field reflecting the vast difference among subjects. However, most algorithms are developed with intended use within the neuroimaging field, e.g., for template generation, template guided segmentation, quantity group difference by registering subjects' images to the same template (Oliveira and Tavares 2014; Toga and Thompson 2001). When applying for mesh morphing, a higher requirement is imposed on the smoothness of the resulting displacement field than usually required in the neuroimaging field, not only associated with FE models' runnability but also prediction accuracy. For the developed hierarchical registration pipeline in this study, both the choice of Demons (Vercauteren et al. 2009) and Dramms algorithms (Ou et al. 2011), as well as the hierarchical design are essential to obtain displacements fields that allow generating subject-specific head models with competitive personalization accuracy, meanwhile with satisfactory element quality without mesh repairing.

Demons registration allows morphing brains with large differences; however, it tends to result in displacement fields that may lead to excessive element distortion according to our previous experience (Li et al. 2013; von Holst and Li 2013; von Holst et al. 2012). The hierarchical design of the pipeline is to utilize Demons' capacity for handling large shape differences by performing Demons registration as the first step with binary cranial masks as input that allows obtaining a displacement field reflecting well overall cranial shape (mean DICE of 0.975, Fig. 7). Dramms registration is performed in the 2nd step on the skull stripped T1W images inherited from the 1 st step. The focus of the 2 nd step registration is to align local brain structures and CSF (outer CSF and ventricles) by using brain MRI information without the need for segmentation. The choice of Dramms is based on its promising performance due to its advancing in hierarchical attribute matching mutual-saliency mechanism (Ou et al. 2011). It will be interesting to investigate if other nonlinear algorithms would achieve good performance for the detailed brain or not, such as B-spline and Burr's elastic previously used for morphing head models with smooth brains (Ji et al. 2015; Wu et al. 2019). Besides, other popular algorithms developed in the neuroimaging field (e.g., DRATEL by Ashburner (2007), and more found in Ou et al. (2014)) when used alone or replacing Dramms in the 2nd step may result in better performance or not. Nevertheless, the proposed hierarchical registration pipeline leads to competitive performance in generating detailed subject-specific head models with satisfactory element quality without the need for further element repairing, meanwhile achieves DICE values 
comparable to or higher than that in the neuroimaging field (Ou et al. 2014).

With the established pipeline, new advanced registration algorithms developed in the neuroimaging field can be readily implemented to generate subject-specific models reflecting even better the intersubject-variability for both brain and WM fiber morphologies. The hierarchical image registration pipeline is not only applicable for this current ADAPT head model but also can be used to morph other head models as a baseline, such as smoothed voxel head models. Further, due to its capacity for handling highly nonlinear warping, the pipeline can also be used to generate models with pathologies with brain structural changes such as decompressive craniotomy when the brain is expanding outside the skull (von Holst et al. 2012).

The proposed pipeline allows good alignment for local brain regions such as $\mathrm{CC}$, achieving almost perfect alignment (DICE of 0.95) for CC for the smallest female, though less ideal (DICE of 0.85) for the largest female (Fig. 3). Note that the less ideal DICE values for some cases are not to be seen as performance indicators for the Dramms algorithm per se. Since the performance relies on input image alignment for Dramms, which can be tuned to achieve better performance. Nevertheless, the DICE values for GM and WM are difficult to improve by registration. This issue could be improved by regrouping WM according to the registered neuroimages which will allow representing the subject's WM accurately. Therefore, combining regrouping would allow the mesh morphing approach as an efficient approach for generating subject-specific models with competitive accuracy comparing with developing a model from scratch. For example, the Hexotic approach as described for the baseline model requires accurate image segmentation, generating surfaces, manual generation of membrane elements, which is a tedious process.

\subsection{Brain-skull relative motion validation performance comparing with previous models}

Regarding model validation performance on brain-skull relative motion, the ADAPT head model shows consistently higher CORA scores comparing with the original KTH head model, while has comparable CORA scores with the KTHFSI model and the KTH detailed head model. The seven cases for brain-skull relative motion validation selected here are because they consistently have a duration longer than $40 \mathrm{~ms}$. Besides, only reliable NDTs are included in CORA score calculation, justifying the same weight factor used for all NDTs (i.e., CORA score for each case is a mean of NDT curves). CORA scores from previous models are either newly calculated or recalculated in this study to ensure all values are directly comparable among models. In fact, CORA scores for the original KTH head model for
C288-T3, C380-T4, C380-T6, C393-T3 have been presented earlier, showing comparable CORA scores with THUMS, GHBMC, see Table 8 in the study by Giordano and Kleiven (2016). Note despite this study followed the same CORA calculation method (with corridor method excluded) and used recommended global settings same as proposed by Giordano and Kleiven (2016), the same weighting factor is used for all the evaluated NDTs instead of using the proposed weights for each NDTs as listed in Table 5 (Giordano and Kleiven 2016). The reason is that seems no consensus has been reached among the research community on the proposed weighting factors and using equal weight for all NDTs is justified by only include reliable NDTs as plotted to allow an easier comparison for future studies. Nevertheless, the CORA scores for the overlapping cases are close between the two studies, e.g., for C380-T4 5.26 shown in Table 8 in Giordano and Kleiven (2016), close to 0.551 reported here (note differing by a factor of 10 in the calculation).

\subsection{Experimental brain strain for head model validation}

Regarding experimental brain strain data used for model validation, the cluster brain strain presented in Zhou et al. (2019b) is used, which is recalculated based on the original brain-skull relative motion experimental data from Hardy et al. (2007) using a tetra approach instead of a triad approach used in the original study (Hardy et al. 2007). Though it's well recognized (Zou et al. 2007; Zhao and Ji 2020) and recently has been extensively verified (Zhou et al. 2019b, 2018) that a model validated against brain-skull relative motion may not necessarily guarantee its strain prediction accuracy. Therefore, it's suggested that a head model with the intended use for strain prediction should be validated against experimental brain strain data. However, despite the availability of the strain data presented in Hardy et al. (2007) along with the brain motion data, it's seldomly used for head model validation in contrast to motion data being widely used. This may be partially attributed to concerns on the quality of strain data, especially the initially reported $\sim 2-5 \%$ peak strains are rather low for an injurious impact (see a detailed discussion by Zhao and Ji (2020) and references therein).

To address the quality concerns on strain data originally presented in Hardy et al. (2007), a two-step effort has been undertaken recently. As a first step, Zhou et al. (2018) reanalyzed and updated the brain strain data using the same triad approach and developed three criteria to assess the eligibility of the NDT clusters suitable for strain calculation. As a second step, a tetra approach is used for brain strain calculation (Zhou et al. 2019b), reflecting better the 3D experimental brain deformation than the triad approach. Note the tetra or triad approach is just one of another way to estimate brain strain 
from NDT motion, alternative approach has been proposed also, e.g., a generalized marker-based strain sampling approach to estimate and compare regional strains (Zhao and Ji 2020). Indeed, experimental brain strain data calculated by the tetra approach are much larger than by the triad approach, indicating the original triad strains (Hardy et al. 2007), also the reanalyzed triad strains (Zhou et al. 2018) largely underestimated the experimental brain strain. Thus, the experimental brain strain data calculated by the triad approach are not recommended to be used for head model validation due to its large underestimation of the real brain strain in the experiment.

Note that for nearly incompressible material as the brain, shear strains should be close to principal strains, indeed as the ADAPT model predicted for all the seven clusters (see Fig. 6 simulated shear strain and principal strain). However, unexpected large differences are observed between the experimental principal strain and experimental shear strain (Fig. 6). However, according to a study by Bradshaw (2003), it is quantified that for brain tissue, the principal strain should fall within the range of $2 / 3$ to $4 / 3$ of the maximum shear strain based on the theoretical interpretation, while when reanalyzing the experimental strain data from Zhou et al. (2019b), of all the 15 clusters, the experimental principal strain consistently falls within the shear strain band, correlating with the theoretical finding by Bradshaw (2003). Such correlation seems to indicate an acceptable quality of the 15 cluster brain strain dataset including the 7 used here for validation. Therefore, the recalculated cluster brain strains using the tetra approach have been verified and justified thoroughly (Zhou et al. 2019b), and can be taken as experimental brain strain for validating brain strain response of FE head models.

\subsection{Brain strain validation performance}

Seven cluster strains are chosen (out of 15 in total) for model validation as they consistently have a duration of over $40 \mathrm{~ms}$. The shape and phase show a good match between ADAPT model-simulated and experimental brain strain, but a large discrepancy in magnitude is observed in most clusters (Fig. 6). Further, the simulated brain strains are consistently lower than the experimental strain in all the evaluated clusters except for C380-T1 C1. It will be interesting to study more clusters, especially, experimental strains for C241-T5 C2, C241-T6 C2, C393-T2 C1 have much lower peak strains (all <0.1) (Zhou et al. 2019b). It's expected that for some of these clusters, simulated brain strain may be larger than experimental strain, which, however, is yet to be performed. This also reminds one important difference compared with NDT motion data (many NDT curves even for one case, e.g. 14 NDTs and each has XYZ displacements), that choosing only several or certain cluster strains may lead to a biased impression that the simulated brain strain too low compared with experimental data; this further reminds that caution should be taken when tuning material parameters to satisfy brain strain performance when only use a limited number of clusters (as shown in Fig. 6 for the seven clusters selected in this study consistently shows lower strain in the model, but may not be the case when more cluster are evaluated as discussed above).

Therefore, the large peak discrepancy between ADAPT and experimental cluster strain is suggested not to be seen as a concern for the model performance, rather it opens a question not specific to this model: How to use the experimental cluster strain for validation for the models that chose to use this strain data. Especially, how to evaluate model performance when only a few cluster curves are available versus NDT motion curves where are many. Further, how to extract brain strain for a model with coarse mesh. Note CORA scores for brain strain are actually higher than brain motion despite large magnitude discrepancy, which seems to remind CORA scores may not be a proper index, which thus raises a question on how to weight between NDT motion and strain to reach an overall biofidelity ranking for a model.

The recalculated experimental cluster brain strain data are a result of the two-step efforts (Zhou et al. 2018, 2019b) attempted to best utilize the NDT motions close to or at injury level originally measured by Hardy et al. (2007). The recalculated experimental cluster brain strain data provide a possibility to evaluate head models' brain strain prediction capacity; however, there are challenges, as mentioned above, that need integrated effort among the research community to allow its proper use for head model validation. Efforts toward this direction have been initiated (Zhao and Ji 2020), and a thorough discussion on the use of strain data for model validation and the insufficiency of CORA scores for model biofidelity rating can be found therein.

\subsection{Material modeling choice for the brain, falx, tentorium, dura, and pia}

The ADAPT model uses the same material properties for the brain as the previously validated KTH head model (Kleiven 2007) with a coarse brain mesh. Indeed, studies have demonstrated that models with finer meshes would lead to the prediction of larger brain-skull relative motions and larger brain strains with the same set of material properties (Giudice et al. 2019; Zhao and Ji 2019a, b). It is thus suggested that it may not be appropriate to adopt material properties of the brain obtained from a coarse mesh to a model with a much finer mesh, and vice versa (Zhao and Ji 2019a, b). This is indeed a valid concern especially if brain parameters were obtained by adjusting/optimization to satisfy model validation, resulting in model-specific material properties that may not be directly translatable to other models, especially with largely different brain mesh sizes. Keeping this in mind, it seems questionable to adopt brain material properties from the KTH head model to ADAPT. However, note that the brain 
material properties presented in (Kleiven 2007) were based on careful analysis and data fitting of experimental data, thus can be considered as model-independent and translatable to the ADAPT model, while updating falx/tentorium material properties with a nonlinear model instead of using the same linear elastic model (Young's modulus of $31.5 \mathrm{MPa}$ ) is based findings from a recent study (Ho et al. 2017), showing the importance of using nonlinear material models that allow reflecting the nonlinear properties falx/tentorium as shown from experiments. Similarly, pia mater is updated in the ADAPT model using a nonlinear model based on experimental data. Due to significant differences in mesh sizes, updated material models for pia/falx/tentorium/dura, as well as sliding contact in the KTH head model versus continuous mesh in ADAPT, different brain responses are expected from the two models under the same loadings. Indeed, the ADAPT model, in general, has a larger brain-skull relative motion for most NDTs (see Figs. 15, 16 and 17), and has higher CORA scores for the seven cases than the KTH head model (Table 3). Further, the detailed brain morphology (sulci/gyri) included in the current model may potentially alter brain tissue response, considering the CSF penetrates and pia mater holding the brain at the sulci/gyri comparing with models with smooth brain surface. Nevertheless, a more systematic investigation is required to study the potential prediction differences between ADAPT and the original KTH head model. Consequently, injury criteria developed (Kleiven 2007) may not be directly applicable to the ADAPT model, and care should be taken when using the ADAPT model for injury prediction based on existing injury criteria developed from the KTH head model and other head models.

Mechanically anisotropic brain tissue models, in particular, the Gasser, Ogden and Holzapfel $(\mathrm{GOH})$ model have been implemented in FE head models (Giordano et al. 2014; Giordano and Kleiven 2014b; Giordano et al. 2017; Zhao and Ji 2019b), by connecting anisotropic diffusion properties from DTI to mechanical anisotropy (Giordano and Kleiven 2014a). Some experimental studies suggest that brain tissue is mechanically isotropic, e.g., Budday et al. (2017), while others suggest brain tissue show significant directional trends, e.g., Prange and Margulies (2002), Feng et al. (2017). More discussion on the controversies can be found in a review study by Budday et al. (2019). Nevertheless, both isotropic and anisotropic models are being used in head models for studying TBIs; see review studies (Giudice et al. 2019; Madhukar and Ostoja-Starzewski 2019). In the current study, the isotropic hyperviscoelastic material model is used for brain tissue, and axonal strains are calculated by projecting strain tensors to WM fiber tract directions as earlier studies have shown MAS as a potentially improved predictor for brain injury (Sullivan et al. 2015; Zhao et al. 2017, 2016) using the same approach or explicitly model brain as GOH (Giordano and Kleiven 2014b). Nevertheless, how brain material difference (e.g., isotropic versus anisotropic) may influence brain strain in an anatomically detailed head model is yet to be studied.

\subsection{Limitation and future work}

There are some limitations in the ADAPT model to be noted. The ADAPT model has a conforming mesh at all interfaces between the entire brain and CSF (i.e., outer CSF-brain/dura, ventricle-brain). Still, non-smooth boundaries exist at brain subregions, including GM and WM interface. The subregions of the brain and diploe porous skull bone are grouped according to the spatial correspondence with the segmented images via an automatic script, which seems often similarly practiced in other head models as well, e.g., WM meshes manually picked according to geometry data in the GHBMC (Mao et al. 2013a), and the same for the KTH head model (Kleiven 2007). Despite the non-smoothness, strain concentration is often not a concern, especially if using the same material properties for the entire brain as done in this study. Further, a continuous mesh is used throughout the ADAPT model, which can be further improved by including FSI at the brain-ventricle interface as done earlier (Zhou et al. 2020), and further to implement FSI at the brain-skull interface (Zhou et al. 2019a) in the future, despite a technical challenge to implement FSI on the complex sulci and gyri than a smooth brain model. Further, the model doesn't validate well with the chosen in vivo data, and future investigations are needed to improve the model's capacity for predicting brain response under non-injurious low impact loading. The ADAPT model also has a substantially longer simulation runtime compared with models with fewer elements. Finally, the results presented in Sects. 3.4, 3.5, 3.6 are to be seen as parametrical studies based on a validated baseline model, highlighting the differences in brain strain prediction among individuals under the same impact loading condition.

\section{Conclusion}

This study presents the development of an anatomically detailed head model with conforming hexahedral mesh (the ADAPT head model) equipped with a hierarchical image registration pipeline for efficient generation of subject-specific models by mesh morphing. The model is validated against brain-skull relative, brain strain, and intracranial pressure, showing comparable performance with previous models. The six-subject specific head models generated using the ADAPT model and the pipeline demonstrate the capacity of the ADAPT model and the pipeline for a fast generation of anatomically detailed subject-specific head models with largely varying brain sizes/shapes with competitive personalization accuracy on capturing individual's brain structures. 
The simulation results show significant differences in brain strain and axonal strain, motivating the necessity for using subject-specific head models for evaluating brain injuries. The ADAPT model due to its uniqueness of the complete ventricular systems, including 3rd and 4th ventricles in connection with outer CSF via aqueduct, together with conforming meshed sulci and gyri and subject-specific WM fibers, could potentially provide new insights into TBI mechanisms. The verified performance of the ADAPT head model equipped with the personalization approach addresses the challenges in subject-specific FE model generation, opening an opportunity for studying personalized brain responses, as well as developing personalized head protection systems. The research community may find the hierarchical registration pipeline useful to morph other anatomically detailed head models, such as smoothed-voxel head models.

Acknowledgements Open access funding provided by Royal Institute of Technology. The authors would like to acknowledge the earlier version of the Python code from Chiara Giordano, which was adapted and used in this study. Our special thanks go to Dr. Yangming Ou and Dr. Steve Pieper, who generously spent their time advising Dramms registration, and to Dr. Loïc Maréchal who provided great support for the usage of the software Hexotic. The authors thank the three anonymous reviewers and Prof. Peter J. Hunter for their stimulating comments and valuable suggestions that substantially improved this paper. The WUM $\mathrm{HCP}$ data of the six subjects used in this study were provided [in part] by the Human Connectome Project, WU-Minn Consortium (Principal Investigators: David Van Essen and Kamil Ugurbil; 1U54MH091657) funded by the $16 \mathrm{NIH}$ Institutes and Centers that support the NIH Blueprint for Neuroscience Research; and by the McDonnell Center for Systems Neuroscience at Washington University. The computations were performed on resources provided by the Swedish National Infrastructure for Computing (SNIC) at Center for High Performance Computing (PDC) at KTH. Gong Jing at KTH PDC center is acknowledged for assistance for the installation of needed software on the PDC resources. The present study was supported by research funds from KTH-Royal Institute of Technology, Stockholm, the Swedish Research Council Grants (No. 2016-04203 and No. 2016-05314), and European Union's Horizon 2020 research and innovation program under the Marie Curie Grant Agreement No. 642662.

\section{Compliance with ethical standards}

Conflict of interest The authors declare that they have no conflict of interest.

Open Access This article is licensed under a Creative Commons Attribution 4.0 International License, which permits use, sharing, adaptation, distribution and reproduction in any medium or format, as long as you give appropriate credit to the original author(s) and the source, provide a link to the Creative Commons licence, and indicate if changes were made. The images or other third party material in this article are included in the article's Creative Commons licence, unless indicated otherwise in a credit line to the material. If material is not included in the article's Creative Commons licence and your intended use is not permitted by statutory regulation or exceeds the permitted use, you will need to obtain permission directly from the copyright holder. To view a copy of this licence, visit http://creativecommons.org/licenses/by/4.0/.

\section{Appendix 1}

\section{Personalization accuracy and mesh quality of the personalized models by morphing}

The baseline T1W image (Fig. 13a) is warped to a personalized T1W image for each subject (Fig. 13b) using the procedure presented in Sect. 2.4. The personalized T1W image is paired with the subject-specific mesh. Thus a comparison between it and the subject's own T1W (Fig. 13c) (the ground truth) allows evaluating how the subject-specific model reflects the subject's head geometry. The results show not only the overall head shape but also internal brain structures are well correlated (Fig. 13b, c). The personalized T1W images (Fig. 13b) and subject T1W (Fig. 13c) are then segmented for the cranial mask, brain, and seven local brain regions illustrated in Fig. 4 in the main text for DICE calculation.

DICE coefficient for the six subjects corresponding to the boxplot Fig. 7 in the main text is listed in Table 6 .

\section{Appendix 2}

\section{Brain-skull relative motion and brain strain validation comparing with previous head models}

Brain-skull relative motion for three cases predicted from the ADAPT, and the original KTH head model (Kleiven 2007) are compared for C288-T3 (Fig. 15), C380-T1 (Fig. 16), C380-T2 (Fig. 17), together with experimental data from (Hardy et al. 2007). Note that C288-T3 NDT8 has a duration of $39 \mathrm{~ms}(<40 \mathrm{~ms})$. Thus NDTs from 1 to 14 except NDT8 are included in the plot also in the CORA calculation. Besides, for the six impacts delivered to subject C380 (i.e., C380-T1 to T6), the motion of NDT9 failed to exhibit a motion trend toward its starting position (details found in (Zhou et al. 2018). Thus NDT1-14 except NDT9 are included in the plot also in the CORA calculation. Finally, for C393-T3, NDT13, all the 14 NDTs (NDT1 to 14) are included in the plot also in the CORA calculation.

CORA scores calculated for the KTH detailed head model (Zhou et al. 2019b) on brain strain are presented in Table 8 in comparison with the ADAPT head model presented in Table 4 in the main text. 


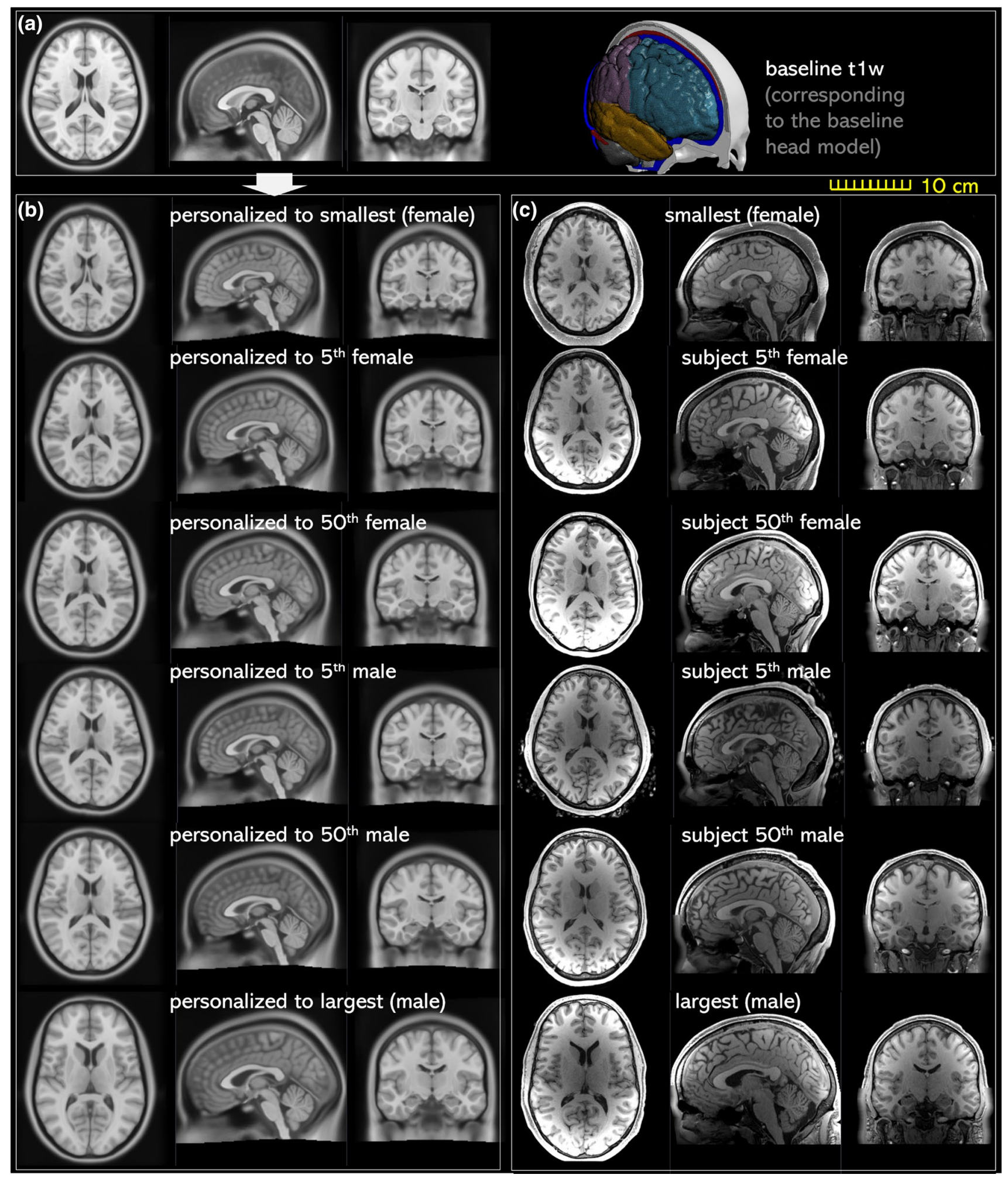

Fig. 13 Baseline T1W image a and personalized T1W image (left column) comparing with the ground truth of the T1W image of the subject. Transverse, sagittal, and coronal cross sections are captured for each image 
Table 6 DICE coefficients for the six subjects

\begin{tabular}{lllllllllll}
\hline Subject ID & Cranial & Brain & GM & WM & Cerebellum & Hipp & Thal & CC & BS & LV \\
\hline 118,124 & 0.97 & 0.92 & 0.66 & 0.76 & 0.91 & 0.75 & 0.90 & 0.95 & 0.85 & 0.72 \\
172,635 & 0.98 & 0.90 & 0.59 & 0.71 & 0.93 & 0.80 & 0.89 & 0.81 & 0.80 & 0.67 \\
185,038 & 0.98 & 0.90 & 0.58 & 0.70 & 0.90 & 0.81 & 0.89 & 0.89 & 0.78 & 0.78 \\
223,929 & 0.97 & 0.90 & 0.57 & 0.71 & 0.93 & 0.76 & 0.85 & 0.85 & 0.83 & 0.77 \\
568,963 & 0.98 & 0.89 & 0.59 & 0.69 & 0.90 & 0.76 & 0.90 & 0.93 & 0.85 & 0.68 \\
771,354 & 0.97 & 0.89 & 0.56 & 0.69 & 0.87 & 0.68 & 0.86 & 0.90 & 0.69 & 0.62 \\
Average & 0.975 & 0.90 & 0.59 & 0.71 & 0.91 & 0.76 & 0.88 & 0.88 & 0.80 & 0.71 \\
\hline
\end{tabular}

Table 7 Element quality of the ADAPT model and the six subject-specific head models generated by morphing

\begin{tabular}{|c|c|c|c|c|c|c|c|c|c|c|c|c|}
\hline \multirow[t]{3}{*}{ Head model } & \multicolumn{12}{|c|}{ Element quality Index } \\
\hline & \multicolumn{2}{|l|}{$\begin{array}{l}\text { Jacobian } \\
\geq 0.5\end{array}$} & \multicolumn{2}{|l|}{$\begin{array}{l}\text { Warpage }\left({ }^{\circ}\right) \\
\leq 30\end{array}$} & \multicolumn{2}{|l|}{$\begin{array}{l}\text { Skew }\left({ }^{\circ}\right) \\
\leq 60\end{array}$} & \multicolumn{2}{|l|}{$\begin{array}{l}\text { Aspect ratio } \\
\leq 8\end{array}$} & \multicolumn{2}{|c|}{$\begin{array}{l}\text { Min. angle }\left({ }^{\circ}\right) \\
\geq 30\end{array}$} & \multicolumn{2}{|c|}{ Max. angle $\left({ }^{\circ}\right) \leq 150$} \\
\hline & Percent & Min. & Percent $(\%)$ & Max. & Percent (\%) & Max. & Percent (\%) & Max. & Percent $(\%)$ & Min. & Percent $(\%)$ & Max. \\
\hline ADAPT & 98 & 0.45 & 92 & 111.76 & 99.9 & 69.95 & 100 & 6.62 & 99.8 & 17.98 & 99.9 & 161.94 \\
\hline \multicolumn{13}{|c|}{ Personalized models } \\
\hline Smallest (female) & 95 & 0.27 & 91 & 134.24 & 99.9 & 70.75 & 100 & 7.83 & 99.9 & 13.65 & 98 & 168.00 \\
\hline 5th perc. female & 95 & 0.28 & 91 & 117.11 & 99.9 & 70.61 & 99.9 & 8.64 & 99.9 & 11.65 & 98 & 168.70 \\
\hline 50th perc. female & 94 & 0.21 & 91 & 121.79 & 99.9 & 74.48 & 100 & 7.79 & 99.0 & 12.24 & 98 & 203.35 \\
\hline 5th perc. male & 95 & 0.32 & 91 & 125.85 & 99.9 & 69.22 & 99.9 & 9.05 & 99.9 & 14.57 & 98 & 168.33 \\
\hline 50th perc. male & 95 & 0.26 & 92 & 114.11 & 99.9 & 70.40 & 99.9 & 8.20 & 99.9 & 11.54 & 98 & 170.14 \\
\hline Largest (male) & 95 & 0.31 & 92 & 110.42 & 99.9 & 73.14 & 100 & 7.38 & 99.9 & 14.27 & 98 & 169.01 \\
\hline
\end{tabular}
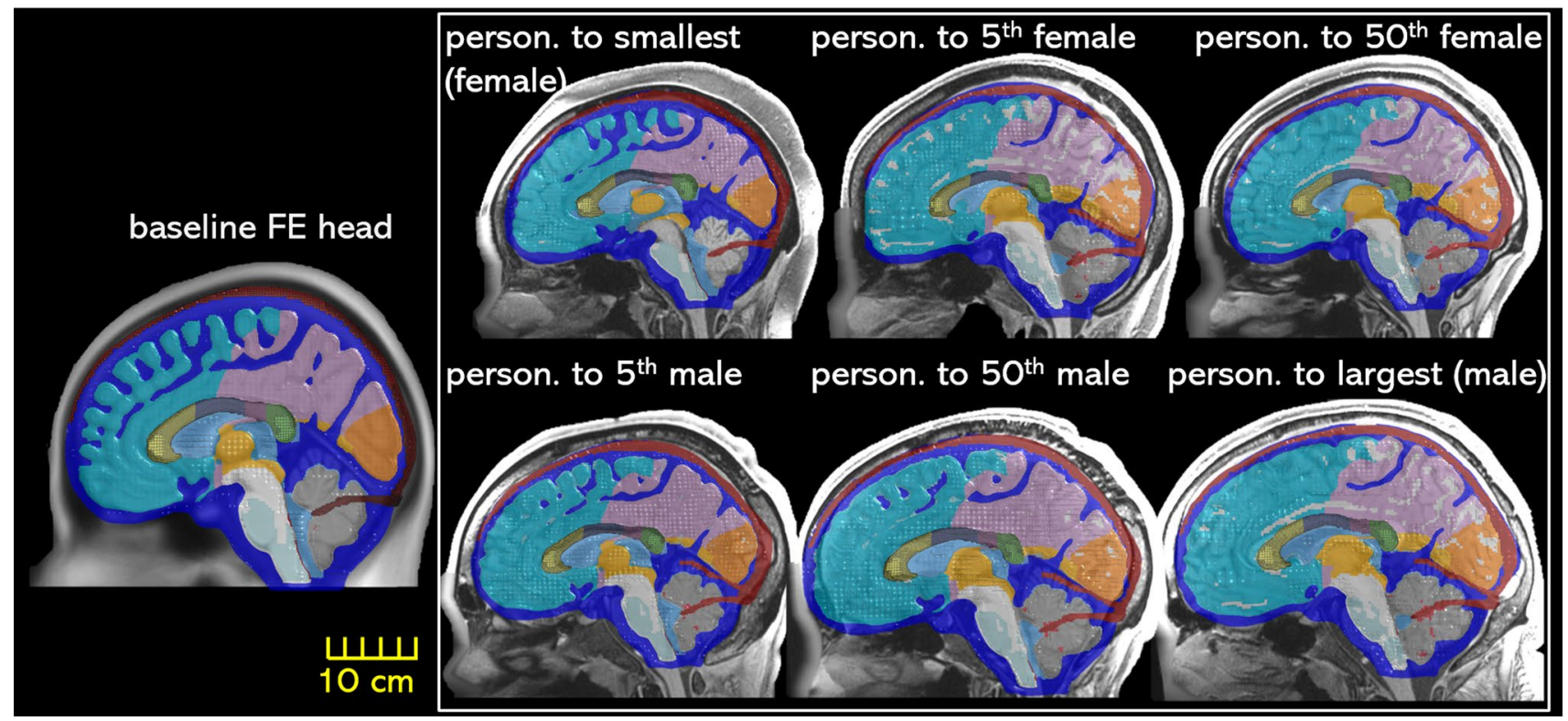

Fig. 14 FE mesh of the six generated subject-specific head models by morphing. Sagittal section of the six head FE mesh is overplayed with the subject's T1W image. The mesh is made half transparent to show the correspondence between the mesh and the ground truth T1W image 

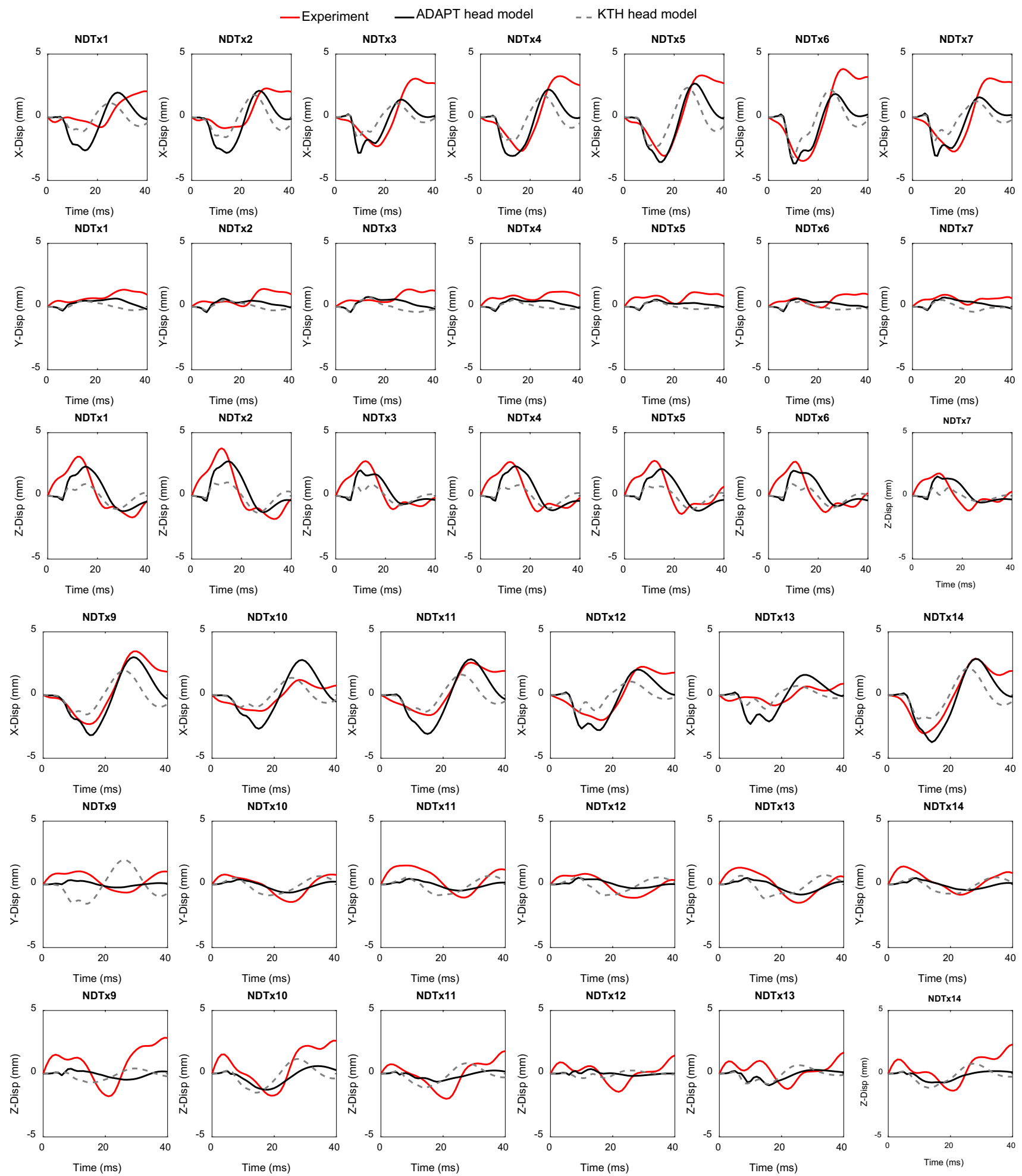

Fig. 15 Comparison between experimental and simulated brain-skull relative motion by the ADAPT head model and KTH head model (Kleiven 2007) for the experiment C288-T3 (Hardy et al. 2007) 

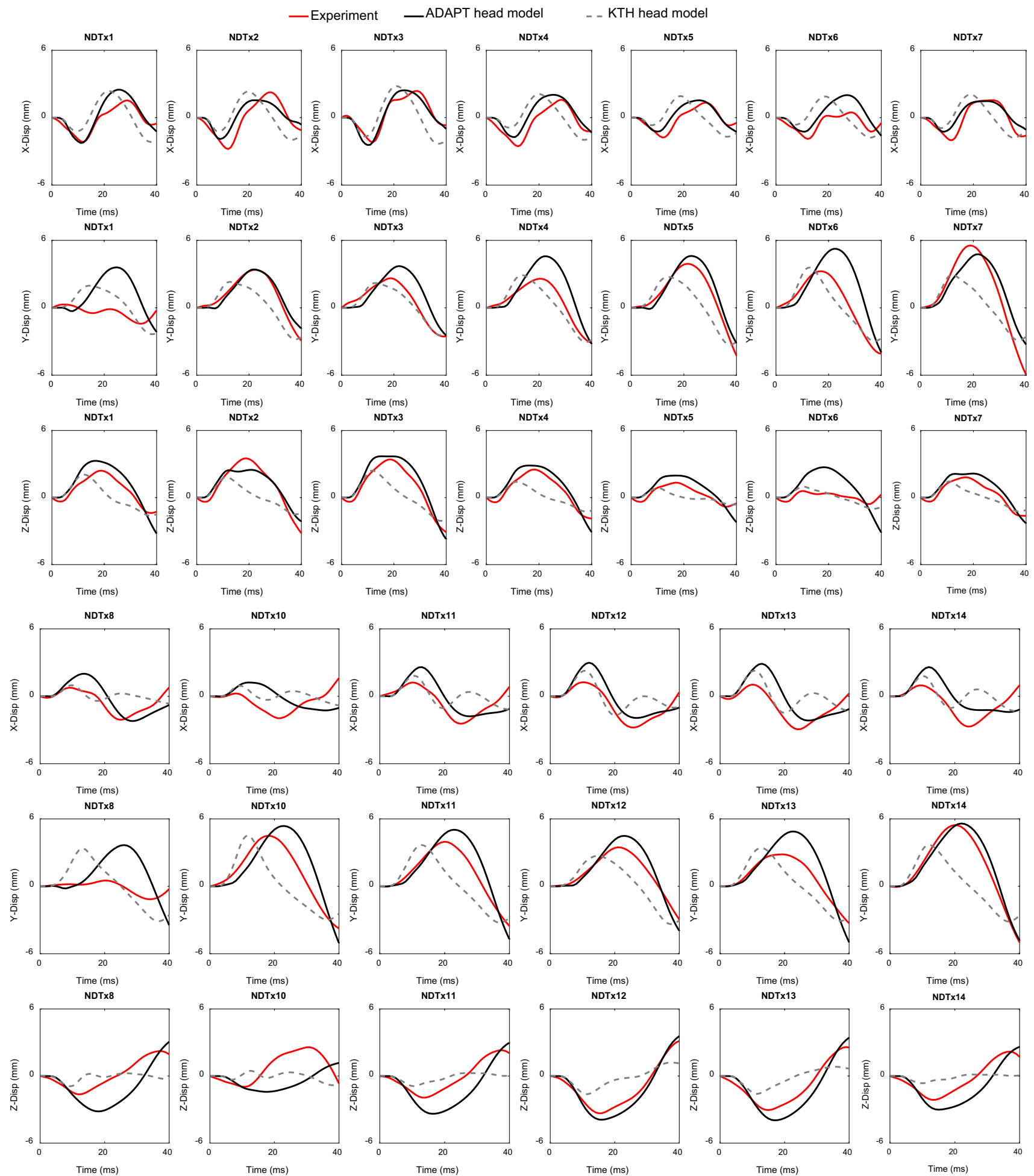

Fig. 16 Comparison between experimental and simulated brain-skull relative motion by the ADAPT head model and KTH head model (Kleiven 2007) for the experiment C380-T1 (Hardy et al. 2007) 

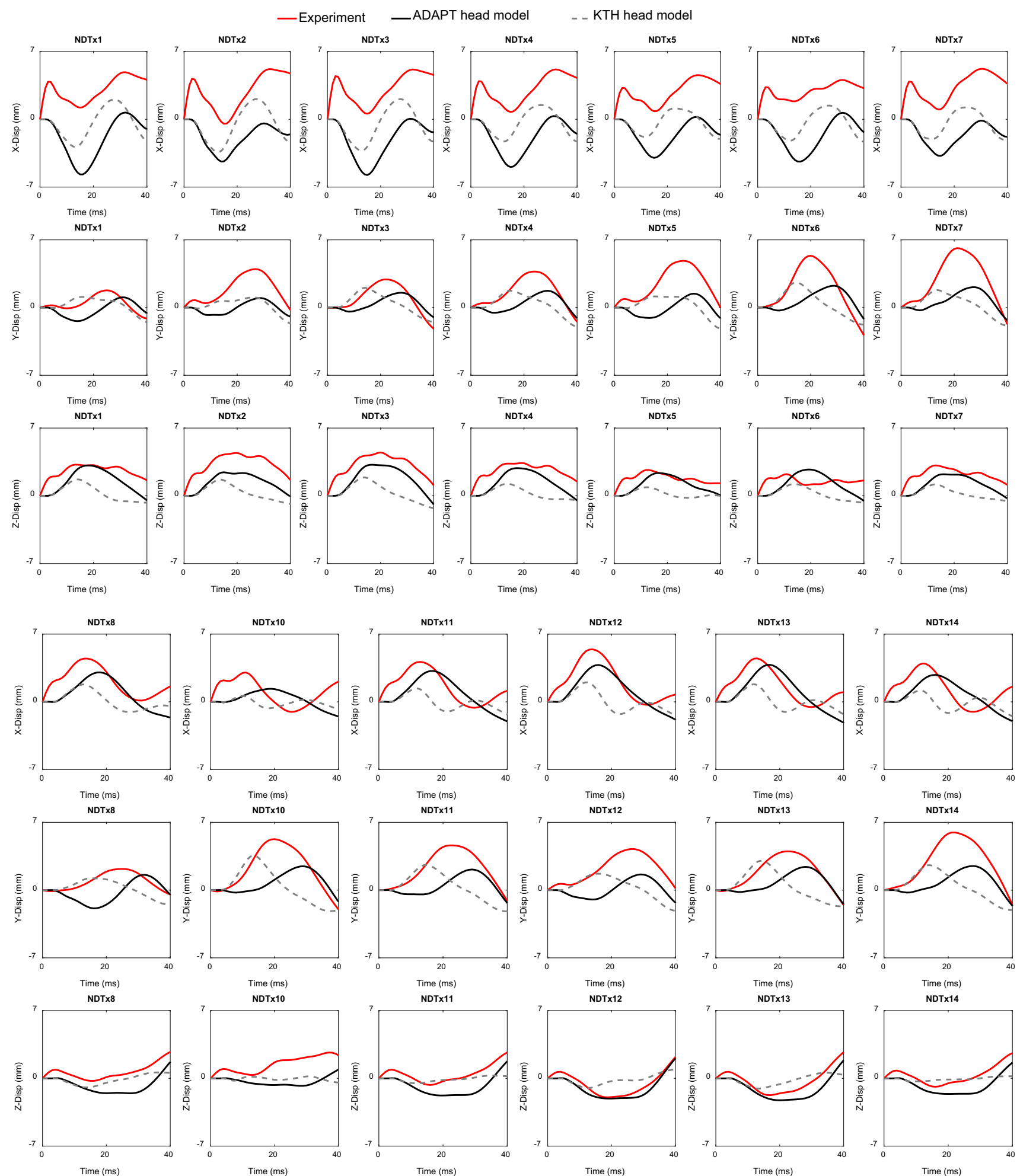

Fig. 17 Comparison between experimental and simulated brain-skull relative motion by the ADAPT head model and KTH head model (Kleiven 2007) for the experiment C380-T2 (Hardy et al. 2007) 
Table 8 CORA scores of the KTH detailed head model (Zhou et al. 2019b) on principal and shear strain

\begin{tabular}{|c|c|c|c|c|c|c|c|c|}
\hline \multirow{2}{*}{$\begin{array}{l}\text { Exp. cases } \\
\text { (Zhou et al. 2019b) }\end{array}$} & \multicolumn{4}{|c|}{ Principal strain } & \multicolumn{4}{|c|}{ Shear strain } \\
\hline & $V$ (shape) & $G($ size $)$ & $P$ (phase) & CORA score & $V$ (shape) & $G($ size $)$ & $P$ (phase) & CORA score \\
\hline C288-T3 C1 & 0.929 & 0.202 & 0.797 & 0.643 & 0.954 & 0.229 & 0.919 & 0.701 \\
\hline C380-T1 C1 & 0.981 & 0.843 & 0.797 & 0.874 & 0.975 & 0.656 & 0.919 & 0.850 \\
\hline C380-T2 C1 & 0.993 & 0.286 & 0.999 & 0.760 & 0.986 & 0.409 & 0.999 & 0.798 \\
\hline C380-T3 C1 & 0.982 & 0.239 & 0.999 & 0.740 & 0.982 & 0.301 & 0.919 & 0.734 \\
\hline C380-T4 C1 & 0.950 & 0.218 & 0.919 & 0.696 & 0.962 & 0.258 & 0.797 & 0.672 \\
\hline C380-T6 C1 & 0.971 & 0.204 & 0.685 & 0.620 & 0.968 & 0.281 & 0.685 & 0.645 \\
\hline C393-T3 C1 & 0.942 & 0.994 & 0.804 & 0.913 & 0.935 & 0.671 & 0.804 & 0.803 \\
\hline Average & & & & $0.749 \pm 0.111$ & & & & $0.743 \pm 0.076$ \\
\hline
\end{tabular}

CORA scores for the first six cases newly calculated based on curves reported in the original study (as CORA scores were not reported). For case C393-T3, simulation is for this study, with CORA newly calculated

Table 9 MPS for brain regions predicted from the six subject-specific head models

\begin{tabular}{lccccc}
\hline Subject & WM & CC & BS & Hipp & Thal \\
\hline Smallest (female) & 0.4142 & 0.2268 & 0.4650 & 0.3163 & 0.2777 \\
5th perc. female & 0.4347 & 0.2523 & 0.4977 & 0.3769 & 0.2694 \\
50th perc. female & 0.4404 & 0.2320 & 0.4990 & 0.3735 & 0.2632 \\
5th perc. male & 0.4601 & 0.2649 & 0.5095 & 0.3832 & 0.3074 \\
50th perc. male & 0.4857 & 0.2902 & 0.5063 & 0.3931 & 0.3138 \\
Largest (male) & 0.5184 & 0.3183 & 0.5171 & 0.4582 & 0.3111 \\
Max. diff. (\%) & $25.18 \%$ & $40.35 \%$ & $11.19 \%$ & $44.9 \%$ & $19.2 \%$ \\
\hline
\end{tabular}

Table 10 MAS for brain regions predicted from the six subject-specific head models

\begin{tabular}{lccc}
\hline Subject & WM & CC & \multicolumn{1}{l}{ BS } \\
\hline Smallest (female) & 0.2534 & 0.1480 & 0.2201 \\
5th perc. female & 0.3172 & 0.1835 & 0.2631 \\
50th perc. female & 0.3569 & 0.1343 & 0.3148 \\
5th perc. male & 0.3643 & 0.1866 & 0.3361 \\
50th perc. male & 0.4173 & 0.1786 & 0.2689 \\
Largest (male) & 0.4257 & 0.2501 & 0.3938 \\
Max. diff. (\%) & $68.01 \%$ & $86.21 \%$ & $78.89 \%$ \\
\hline
\end{tabular}

\section{Appendix 3}

\section{Brain strains predicted from the six personalized models}

The MPS and MAS for brain regions are presented in Tables 9 and 10, corresponding to the boxplot in Fig. 11 in the main text.

\section{References}

Aimedieu P, Grebe R (2004) Tensile strength of cranial pia mater: preliminary results. J Neurosurg 100:111-114

Ashburner J (2007) A fast diffeomorphic image registration algorithm. Neuroimage 38:95-113

Atsumi N, Nakahira Y, Iwamoto M (2016) Development and validation of a head/brain FE model and investigation of influential factor on the brain response during head impact. Int J Veh Saf 9:1-23

Beckwith JG, Zhao W, Ji S, Ajamil AG, Bolander RP, Chu JJ, McAllister TW, Crisco JJ, Duma SM, Rowson S (2018) Estimated brain tissue response following impacts associated with and without diagnosed concussion. Ann Biomed Eng 46:819-830

Beillas P, Berthet F (2017) An investigation of human body model morphing for the assessment of abdomen responses to impact against a population of test subjects. Traffic Inj Prev 18:S142-S147

Bennett CM, Miller MB (2010) How reliable are the results from functional magnetic resonance imaging? Ann N Y Acad Sci 1191:133-155

Bijar A, Rohan P-Y, Perrier P, Payan Y (2016) Atlas-based automatic generation of subject-specific finite element tongue meshes. Ann Biomed Eng 44:16-34

Boyd SK, Müller R (2006) Smooth surface meshing for automated finite element model generation from 3D image data. J Biomech 39:1287-1295

Bradshaw DRS (2003) Linear wave propagation in traumatic brain injury. Doctor Thesis, University of Southampton

Bradshaw D, Morfey C (2001) Pressure and shear response in brain injury models. In 17th International technical conference on the enhanced safety of vehicles. National Highway Traffic Safety Administration, Amsterdam, Netherlands

Bucki M, Lobos C, Payan Y (2010) A fast and robust patient specific finite element mesh registration technique: application to 60 clinical cases. Med Image Anal 14:303-317

Budday S, Sommer G, Birkl C, Langkammer C, Haybaeck J, Kohnert J, Bauer M, Paulsen F, Steinmann P, Kuhl E (2017) Mechanical characterization of human brain tissue. Acta Biomater 48:319-340

Budday S, Ovaert TC, Holzapfel GA, Steinmann P, Kuhl E (2019) Fifty shades of brain: a review on the mechanical testing and modeling of brain tissue. Arch Comput Methods Eng 27:1187-1230

Camacho DL, Hopper RH, Lin GM, Myers BS (1997) An improved method for finite element mesh generation of geometrically complex structures with application to the skullbase. J Biomech 30:1067-1070 
Castellano-Smith AD, Hartkens T, Schnabel J, Hose DR, Liu H, Hall WA, Truwit CL, Hawkes DJ, Hill DL (2001) Constructing patient specific models for correcting intraoperative brain deformation. International conference on medical image computing and computer-assisted intervention. Springer, Berlin, pp 1091-1098

Chen Y, Ostoja-Starzewski M (2010) MRI-based finite element modeling of head trauma: spherically focusing shear waves. Acta Mech 213:155-167

Couteau B, Payan Y, Lavallée S (2000) The mesh-matching algorithm: an automatic 3D mesh generator for finite element structures. J Biomech 33:1005-1009

Davis ML, Koya B, Schap JM, Gayzik FS (2016) Development and full body validation of a 5 th percentile female finite element model. Stapp Car Crash J 60:509-544

Fedorov A, Beichel R, Kalpathy-Cramer J, Finet J, Fillion-Robin J-C, Pujol S, Bauer C, Jennings D, Fennessy F, Sonka M (2012) 3D Slicer as an image computing platform for the quantitative imaging network. Magn Reson Imaging 30:1323-1341

Feigin VL, Theadom A, Barker-Collo S, Starkey NJ, McPherson K, Kahan M, Dowell A, Brown P, Parag V, Kydd R (2013) Incidence of traumatic brain injury in New Zealand: a populationbased study. Lancet Neurol 12:53-64

Feng Y, Abney TM, Okamoto RJ, Pless RB, Genin GM, Bayly PV (2010) Relative brain displacement and deformation during constrained mild frontal head impact. J R Soc Interface 7:1677-1688

Feng Y, Lee C-H, Sun L, Ji S, Zhao X (2017) Characterizing white matter tissue in large strain via asymmetric indentation and inverse finite element modeling. J Mech Behav Biomed Mater 65:490-501

Fernandez J, Mithraratne P, Thrupp S, Tawhai M, Hunter P (2004) Anatomically based geometric modelling of the musculo-skeletal system and other organs. Biomech Model Mechanobiol 2:139-155

Fonov VS, Evans AC, McKinstry RC, Almli C, Collins D (2009) Unbiased nonlinear average age-appropriate brain templates from birth to adulthood. Neuroimage 47:S102

Fonov V, Evans AC, Botteron K, Almli CR, McKinstry RC, Collins DL, Group BDC (2011) Unbiased average age-appropriate atlases for pediatric studies. Neuroimage 54:313-327

Ghajari M, Hellyer PJ, Sharp DJ (2017) Computational modelling of traumatic brain injury predicts the location of chronic traumatic encephalopathy pathology. Brain 140:333-343

Giordano C, Kleiven S (2014a) Connecting fractional anisotropy from medical images with mechanical anisotropy of a hyperviscoelastic fibre-reinforced constitutive model for brain tissue. J R Soc Interface 11:20130914

Giordano C, Kleiven S (2014b) Evaluation of axonal strain as a predictor for mild traumatic brain injuries using finite element modeling. Stapp Car Crash J 58:29-61

Giordano C, Kleiven S (2016) Development of an unbiased validation protocol to assess the biofidelity of finite element head models used in prediction of traumatic brain injury. Stapp Car Crash J 60:363-471

Giordano C, Cloots R, Van Dommelen J, Kleiven S (2014) The influence of anisotropy on brain injury prediction. J Biomech 47:1052-1059

Giordano C, Zappalà S, Kleiven S (2017) Anisotropic finite element models for brain injury prediction: the sensitivity of axonal strain to white matter tract inter-subject variability. Biomech Model Mechanobiol 16:1269-1293

Giudice JS, Zeng W, Wu T, Alshareef A, Shedd DF, Panzer MB (2019) An analytical review of the numerical methods used for finite element modeling of traumatic brain injury. Ann Biomed Eng 47:1855-1872
Glasser MF, Sotiropoulos SN, Wilson JA, Coalson TS, Fischl B, Andersson JL, Xu J, Jbabdi S, Webster M, Polimeni JR, Van Essen DC (2013) The minimal preprocessing pipelines for the human connectome project. Neuroimage 80:105-124

Hardy WN, Mason MJ, Foster CD, Shah CS, Kopacz JM, Yang KH, King AI, Bishop J, Bey M, Anderst W (2007) A study of the response of the human cadaver head to impact. Stapp Car Crash J 51:17-80

Hernandez F, Wu LC, Yip MC, Laksari K, Hoffman AR, Lopez JR, Grant GA, Kleiven S, Camarillo DB (2015) Six degree-offreedom measurements of human mild traumatic brain injury. Ann Biomed Eng 43:1918-1934

Ho J, Kleiven S (2009) Can sulci protect the brain from traumatic injury? J Biomech 42:2074-2080

Ho J, Zhou Z, Li X, Kleiven S (2017) The peculiar properties of the falx and tentorium in brain injury biomechanics. J Biomech 60:243-247

Horgan TJ, Gilchrist MD (2003) The creation of three-dimensional finite element models for simulating head impact biomechanics. Int J Crashworth 8:353-366

Horstemeyer MF, Panzer MB, Prabhu RK (2019) State-of-the-Art modeling and simulation of the brain's response to mechanical loads. Ann Biomed Eng 47:1829-1831

Hu J, Jin X, Lee JB, Zhang L, Chaudhary V, Guthikonda M, Yang KH, King AI (2007) Intraoperative brain shift prediction using a 3D inhomogeneous patient-specific finite element model. J Neurosurg 106:164-169

Ji S, Ford JC, Greenwald RM, Beckwith JG, Paulsen KD, Flashman LA, McAllister TW (2011) Automated subject-specific, hexahedral mesh generation via image registration. Finite Elem Anal Des 47:1178-1185

Ji S, Zhao W, Ford JC, Beckwith JG, Bolander RP, Greenwald RM, Flashman LA, Paulsen KD, McAllister TW (2015) Group-wise evaluation and comparison of white matter fiber strain and maximum principal strain in sports-related concussion. J Neurotrauma 32:441-454

Kang H-S, Willinger R, Diaw BM, Chinn B (1997) Validation of a 3D anatomic human head model and replication of head impact in motorcycle accident by finite element modeling. In: Proceedings of the 41st stapp car crash conference, Lake Buena Vista, Florida, USA. Society of Automotive Engineers, pp 329-338

Kimpara H, Nakahira Y, Iwamoto M, Miki K, Ichihara K, Kawano S-i, Taguchi T (2006) Investigation of anteroposterior head-neck responses during severe frontal impacts using a brain-spinal cord complex FE model. Stapp Car Crash J 50:509-544

Kleiven S (2007) Predictors for traumatic brain injuries evaluated through accident reconstructions. Stapp Car Crash J 51:81-114

Kleiven S, von Holst H (2002) Consequences of head size following trauma to the human head. J Biomech 35:153-160

Lauret C, Hrapko M, Van Dommelen J, Peters G, Wismans J (2009) Optical characterization of acceleration-induced strain fields in inhomogeneous brain slices. Med Eng Phys 31:392-399

Li X, Kleiven S (2018) Improved safety standards are needed to better protect younger children at playgrounds. Sci Rep 8:1-12

Li X, von Holst H, Kleiven S (2013) Decompressive craniectomy causes a significant strain increase in axonal fiber tracts. J Clin Neurosci 20:509-513

Li X, Sandler H, Kleiven S (2017) The importance of nonlinear tissue modelling in finite element simulations of infant head impacts. Biomech Model Mechanobiol 16:823-840

Li X, Sandler H, Kleiven S (2019) Infant skull fractures: accident or abuse?: Evidences from biomechanical analysis using finite element head models. Forensic Sci Int 294:173-182

Liu S, Beillas P, Ding L, Wang X (2020) Morphing an existing open source human body model into a personalized model for 
seating discomfort investigation. SAE Technical Paper No. 2020-01-0874

Maas AI, Menon DK, Adelson PD, Andelic N, Bell MJ, Belli A, Bragge P, Brazinova A, Büki A, Chesnut RM (2017) Traumatic brain injury: integrated approaches to improve prevention, clinical care, and research. Lancet Neurol 16:987-1048

Madhukar A, Ostoja-Starzewski M (2019) Finite element methods in human head impact simulations: a review. Ann Biomed Eng 47:1832-1854

Mao H, Zhang L, Jiang B, Genthikatti VV, Jin X, Zhu F, Makwana R, Gill A, Jandir G, Singh A (2013) Development of a finite element human head model partially validated with thirty five experimental cases. J Biomech Eng 135:111002

Mao H, Gao H, Cao L, Genthikatti VV, Yang KH (2013) Development of high-quality hexahedral human brain meshes using featurebased multi-block approach. Comput Methods Biomech Biomed Eng 16:271-279

Maréchal L (2009) Advances in octree-based all-hexahedral mesh generation: handling sharp features. In Proceedings of the 18th international meshing roundtable. Springer, pp 65-84

Meaney DF, Morrison B, Dale Bass C (2014) The mechanics of traumatic brain injury: a review of what we know and what we need to know for reducing its societal burden. J Biomech Eng 136:0210081

Miller LE, Urban JE, Stitzel JD (2016) Development and validation of an atlas-based finite element brain model. Biomech Model Mechanobiol 15:1201-1214

Miller LE, Urban JE, Kelley ME, Powers AK, Whitlow CT, Maldjian JA, Rowson S, Stitzel JD (2019) Evaluation of brain response during head impact in youth athletes using an anatomically accurate finite element model. J Neurotrauma 36:1561-1570

Mori S, Zhang J (2006) Principles of diffusion tensor imaging and its applications to basic neuroscience research. Neuron 51:527-539

Mori S, Crain BJ, Chacko VP, Van Zijl PC (1999) Three-dimensional tracking of axonal projections in the brain by magnetic resonance imaging. Ann Neurol 45:265-269

Mori S, Oishi K, Jiang H, Jiang L, Li X, Akhter K, Hua K, Faria AV, Mahmood A, Woods R (2008) Stereotaxic white matter atlas based on diffusion tensor imaging in an ICBM template. Neuroimage 40:570-582

Nahum AM, Smith R, Ward CC Intracranial pressure dynamics during head impact. In: Proceedings of the 21st stapp car crash conference, Warrendale, PA, USA. SAE Technical Paper, pp 337-366

Oliveira FP, Tavares JMR (2014) Medical image registration: a review. Comput Methods Biomech Biomed Engin 17:73-93

Ommaya AK, Hirsch AE, Yarnell P, Harris EH (1967) Scaling of experimental data on cerebral concussion in sub-human primates to concussion threshold for man. In: Society (ed) proceedings of the 11st stapp car crash conference, Warrendale, PA, USA, vol Society of Automotive Engineers. p 670906

Ou Y, Sotiras A, Paragios N, Davatzikos C (2011) DRAMMS: Deformable registration via attribute matching and mutual-saliency weighting. Med Image Anal 15:622-639

Ou Y, Akbari H, Bilello M, Da X, Davatzikos C (2014) Comparative evaluation of registration algorithms in different brain databases with varying difficulty: results and insights. IEEE Trans Med Imaging 33:2039-2065

Panzer MB, Myers BS, Capehart BP, Bass CR (2012) Development of a finite element model for blast brain injury and the effects of CSF cavitation. Ann Biomed Eng 40:1530-1544

Panzer MB, Wood GW, Bass CR (2014) Scaling in neurotrauma: how do we apply animal experiments to people? Exp Neurol 261:120-126

Park G, Kim T, Forman J, Panzer MB, Crandall JR (2017) Prediction of the structural response of the femoral shaft under dynamic loading using subject-specific finite element models. Comput Methods Biomech Biomed Eng 20:1151-1166

Prange MT, Margulies SS (2002) Regional, directional, and agedependent properties of the brain undergoing large deformation. J Biomech Eng 124:244-252

Prange MT, Kiralyfalvi G, Margulies SS (1999) Pediatric rotational inertial brain injury: the relative influence of brain size and mechanical properties. In: Proceedings of the 43rd stapp car crash conference, Warrendale, PA, USA, vol 99SC23. Society of Automotive Engineers

Rowson S, Duma SM, Stemper BD, Shah A, Mihalik JP, Harezlak J, Riggen LD, Giza CC, DiFiori JP, Brooks A et al (2018) Correlation of concussion symptom profile with head impact biomechanics: a case for individual-specific injury tolerance. J Neurotrauma 35(4):681-690

Ruan J, Khalil T, King AI (1994) Dynamic response of the human head to impact by three-dimensional finite element analysis. J Biomech Eng 116:44-50

Sahoo D, Deck C, Willinger R (2014) Development and validation of an advanced anisotropic visco-hyperelastic human brain FE model. J Mech Behav Biomed Mater 33:24-42

Samani A, Bishop J, Yaffe MJ, Plewes DB (2001) Biomechanical 3-D finite element modeling of the human breast using MRI data. IEEE Trans Med Imaging 20:271-279

Sigal IA, Hardisty MR, Whyne CM (2008) Mesh-morphing algorithms for specimen-specific finite element modeling. J Biomech 41:1381-1389

Sullivan S, Eucker SA, Gabrieli D, Bradfield C, Coats B, Maltese MR, Lee J, Smith C, Margulies SS (2015) White matter tract-oriented deformation predicts traumatic axonal brain injury and reveals rotational direction-specific vulnerabilities. Biomech Model Mechanobiol 14:877-896

Takhounts EG, Eppinger RH, Campbell JQ, Tannous RE, Power ED, Shook LS (2003) On the development of the SIMon finite element head model. Stapp Car Crash J 47:107-133

Takhounts EG, Ridella SA, Hasija V, Tannous RE, Campbell JQ, Malone D, Danelson K, Stitzel J, Rowson S, Duma S (2008) Investigation of traumatic brain injuries using the next generation of simulated injury monitor (SIMon) finite element head model. Stapp Car Crash J 52:1-31

Toga AW, Thompson PM (2001) The role of image registration in brain mapping. Image Vis Comput 19:3-24

Trotta A, Clark JM, McGoldrick A, Gilchrist MD, Annaidh AN (2020) Biofidelic finite element modelling of brain trauma: importance of the scalp in simulating head impact. Int J Mech Sci 172:105448

Van Essen DC, Smith SM, Barch DM, Behrens TE, Yacoub E, Ugurbil K, WU-Minn HCP Consortium (2013) The WU-Minn human connectome project: an overview. Neuroimage 80:62-79

van Noort R, Black M, Martin T, Meanley S (1981) A study of the uniaxial mechanical properties of human dura mater preserved in glycerol. Biomaterials 2:41-45

Vercauteren T, Pennec X, Perchant A, Ayache N (2009) Diffeomorphic demons: efficient non-parametric image registration. Neuroimage 45:S61-S72

Viceconti M, Bellingeri L, Cristofolini L, Toni A (1998) A comparative study on different methods of automatic mesh generation of human femurs. Med Eng Phys 20:1-10

von Holst H, Li X (2013) Quantification of stretching in the ventricular wall and corpus callosum and corticospinal tracts in hydrocephalus before and after ventriculoperitoneal shunt operation. J Appl Math 2013:350359

von Holst H, Li X, Kleiven S (2012) Increased strain levels and water content in brain tissue after decompressive craniotomy. Acta Neurochir 154:1583-1593 
Wold S, Esbensen K, Geladi P (1987) Principal component analysis. Chemometr Intell Lab Syst 2:37-52

Wu T, Alshareef A, Giudice JS, Panzer MB (2019) Explicit modeling of white matter axonal fiber tracts in a finite element brain model. Ann Biomed Eng 47:1908-1922

Wu T, Antona-Makoshi J, Alshareef A, Giudice JS, Panzer MB (2020) Investigation of cross-species scaling methods for traumatic brain injury using finite element analysis. J Neurotrauma 37:410-422

Zhang L, Yang KH, Dwarampudi R, Omori K, Li T, Chang K, Hardy WN, Khalil TB, King AI (2001) Recent advances in brain injury research: a new human head model development and validation. Stapp Car Crash J 45:369-394

Zhao W, Ji S (2016) Real-time, whole-brain, temporally resolved pressure responses in translational head impact. Interface Focus 6:20150091

Zhao W, Ji S (2019a) Mesh convergence behavior and the effect of element integration of a human head injury model. Ann Biomed Eng 47:475-486

Zhao W, Ji S (2019b) White matter anisotropy for impact simulation and response sampling in traumatic brain injury. J Neurotrauma $36: 250-263$

Zhao W, Ji S (2020) Displacement-and strain-based discrimination of head injury models across a wide range of blunt conditions. Ann Biomed Eng. https://doi.org/10.1007/s10439-020-02496-y

Zhao W, Ford JC, Flashman LA, McAllister TW, Ji S (2016) White matter injury susceptibility via fiber strain evaluation using whole-brain tractography. J Neurotrauma 33:1834-1847
Zhao W, Cai Y, Li Z, Ji S (2017) Injury prediction and vulnerability assessment using strain and susceptibility measures of the deep white matter. Biomech Model Mechanobiol 16:1709-1727

Zhou Z, Li X, Kleiven S, Shah CS, Hardy WN (2018) A reanalysis of experimental brain strain data: implication for finite element head model validation. Stapp Car Crash J 62:293-318

Zhou Z, Li X, Kleiven S (2019) Fluid-structure interaction simulation of the brain-skull interface for acute subdural haematoma prediction. Biomech Model Mechanobiol 18:155-173

Zhou Z, Li X, Kleiven S, Hardy WN (2019b) Brain strain from motion of sparse markers. Stapp Car Crash J 63:1-27

Zhou Z, Li X, Kleiven S (2020) Biomechanics of periventricular injury. J Neurotrauma 37:1074-1090

Zou KH, Warfield SK, Bharatha A, Tempany CM, Kaus MR, Haker SJ, Wells WM III, Jolesz FA, Kikinis R (2004) Statistical validation of image segmentation quality based on a spatial overlap index1: scientific reports. Acad Radiol 11:178-189

Zou H, Schmiedeler JP, Hardy WN (2007) Separating brain motion into rigid body displacement and deformation under low-severity impacts. J Biomech 40:1183-1191

Publisher's Note Springer Nature remains neutral with regard to jurisdictional claims in published maps and institutional affiliations. 